\title{
Exploring D-xylose oxidation in Saccharomyces cerevisiae through the Weimberg pathway
}

\author{
Lisa Wasserstrom¹, Diogo Portugal-Nunes ${ }^{1,3}$, Henrik Almqvist ${ }^{2}$, Anders G. Sandström, ${ }^{1,4}$ Gunnar Lidén ${ }^{2}$ \\ and Marie F. Gorwa-Grauslund ${ }^{1 *}$
}

\begin{abstract}
Engineering of the yeast Saccharomyces cerevisiae towards efficient D-xylose assimilation has been a major focus over the last decades since D-xylose is the second most abundant sugar in nature, and its conversion into products could significantly improve process economy in biomass-based processes. Up to now, two different metabolic routes have been introduced via genetic engineering, consisting of either the isomerization or the oxido-reduction of $\mathrm{D}$-xylose to D-xylulose that is further connected to the pentose phosphate pathway and glycolysis. In the present study, cytosolic D-xylose oxidation was investigated instead, through the introduction of the Weimberg pathway from Caulobacter crescentus in S. cerevisiae. This pathway consists of five reaction steps that connect D-xylose to the TCA cycle intermediate a-ketoglutarate. The corresponding genes could be expressed in S. cerevisiae, but no growth was observed on D-xylose indicating that not all the enzymes were functionally active. The accumulation of the Weimberg intermediate D-xylonate suggested that the dehydration step(s) might be limiting, blocking further conversion into a-ketoglutarate. Although four alternative dehydratases both of bacterial and archaeon origins were evaluated, D-xylonate accumulation still occurred. A better understanding of the mechanisms associated with the activity of dehydratases, both at a bacterial and yeast level, appears essential to obtain a fully functional Weimberg pathway in S. cerevisiae.
\end{abstract}

Keywords: D-Xylose, Weimberg pathway, Saccharomyces cerevisiae, D-Xylonate dehydratase, Caulobacter crescentus, Iron-sulfur clusters

\section{Introduction}

The pentose sugar D-xylose is an abundant carbon source in nature. It is a dominating part of hemicellulose-in the form of xylan-in hardwoods and agricultural crops, but is also present to a lower extent in softwoods. Several microorganisms are capable of metabolizing D-xylose through isomerization, oxido-reduction or oxidation reactions. Most bacteria, such as Escherichia coli, use the $\mathrm{D}$-xylose isomerization pathway, in which $\mathrm{D}$-xylose is converted to $\mathrm{D}$-xylulose by $\mathrm{D}$-xylose isomerase (XI) (Jeffries 1983). In contrast, the oxido-reduction route, involving a two-step process catalyzed by D-xylose

\footnotetext{
*Correspondence: Marie-Francoise.Gorwa@tmb.Ith.se

1 Division of Applied Microbiology, Department of Chemistry, Lund

University, PO Box 124, 22100 Lund, Sweden

Full list of author information is available at the end of the article
}

reductase (XR) and xylitol dehydrogenase (XDH), is more common in yeasts such as Scheffersomyces stipitis (Du Preez and Prior 1985). In both routes, xylulose kinase (XK) converts D-xylulose into D-xylulose-5-P that can be further metabolized via the pentose phosphate pathway (PPP) and glycolysis (Wang and Schneider 1980). The Weimberg pathway, first described in the bacterium Pseudomonas fragi in 1960s, is a different metabolic route based on D-xylose oxidation (Weimberg 1961). This route that has received increasing attention in the past decade, has been characterized in several prokaryotes, including the freshwater bacterium Caulobacter crescentus and the halophilic archaeon Haloferax volcanii (Johnsen et al. 2009; Stephens et al. 2007). In this metabolic pathway, $\mathrm{D}$-xylose is oxidized to the tricarboxylic acid (TCA) cycle intermediate $\alpha$-ketoglutarate in five enzymatic steps, without carbon loss. D-Xylose is initially oxidized to 
$\mathrm{D}$-xylono- $\gamma$-lactone by a $\mathrm{D}$-xylose dehydrogenase (XylB, encoded by $x y l B$ ), that is further converted to the intermediate $\mathrm{D}$-xylonate by a D-xylono- $\gamma$-lactone lactonase (XylC, encoded by $x y l C$ ). D-Xylonate is then subjected to two dehydration reactions by a $\mathrm{D}$-xylonate dehydratase (XylD, encoded by $x y l D$ ) and a 2-keto-3-deoxy-D-xylonate dehydratase (XylX, encoded by $x y l X$ ), yielding 2-keto-3-deoxy-D-xylonate and then $\alpha$-ketoglutarate semialdehyde. In the final step, the semialdehyde is oxidized by $\alpha$-ketoglutarate semialdehyde dehydrogenase (XylA, encoded by $x y l A$ ) into $\alpha$-ketoglutarate. The entry point of carbon into the central cellular metabolism is thus completely different from both the XI and the XR$\mathrm{XDH}$ pathways (Fig. 1). Heterologous expression of the Weimberg pathway from $C$. crescentus has enabled bacteria such as Pseudomonas putida and Corynebacterium glutamicum to grow on D-xylose as the sole carbon source (Meijnen et al. 2009; Radek et al. 2014). However, to the best of our knowledge, the complete Weimberg pathway has not yet been established in fungal species.

Saccharomyces cerevisiae is an extensively investigated yeast due to its unique features and potential to be applied in biotechnological processes aiming for the production of a large range of chemical compounds. Among others, the production of biofuels (e.g. ethanol, farnesene, isobutanol), pharmaceutical drugs (e.g. artemisinic acid) and carboxylic acids has received large attention from both academia and industry (Abbott et al. 2009; Borodina and Nielsen 2014; Buijs et al. 2013; Paddon et al. 2013). Since S. cerevisiae is not capable of naturally fermenting $\mathrm{D}$-xylose, considerable research efforts have been invested in the engineering and optimization of D-xylose assimilation pathways based on XR-XDH or XI in S. cerevisiae, in particular for the production of ethanol or acetyl-CoA derived products [see reviews by e.g. (Hahn-Hägerdal et al. 2007; Kim et al. 2013; Van Maris et al. 2007)]. In that context, the Weimberg pathway that converts D-xylose to $\alpha$-ketoglutarate that enters the metabolism via the TCA cycle could be relevant for the production of carboxylic acids produced or derived from the TCA-cycle. Potential applications for $\alpha$-ketoglutarate are as a dietary supplement, in medical products, and as a platform chemical for the synthesis of heterocyclic compounds (Otto et al. 2011). Importantly, $\alpha$-ketoglutarate

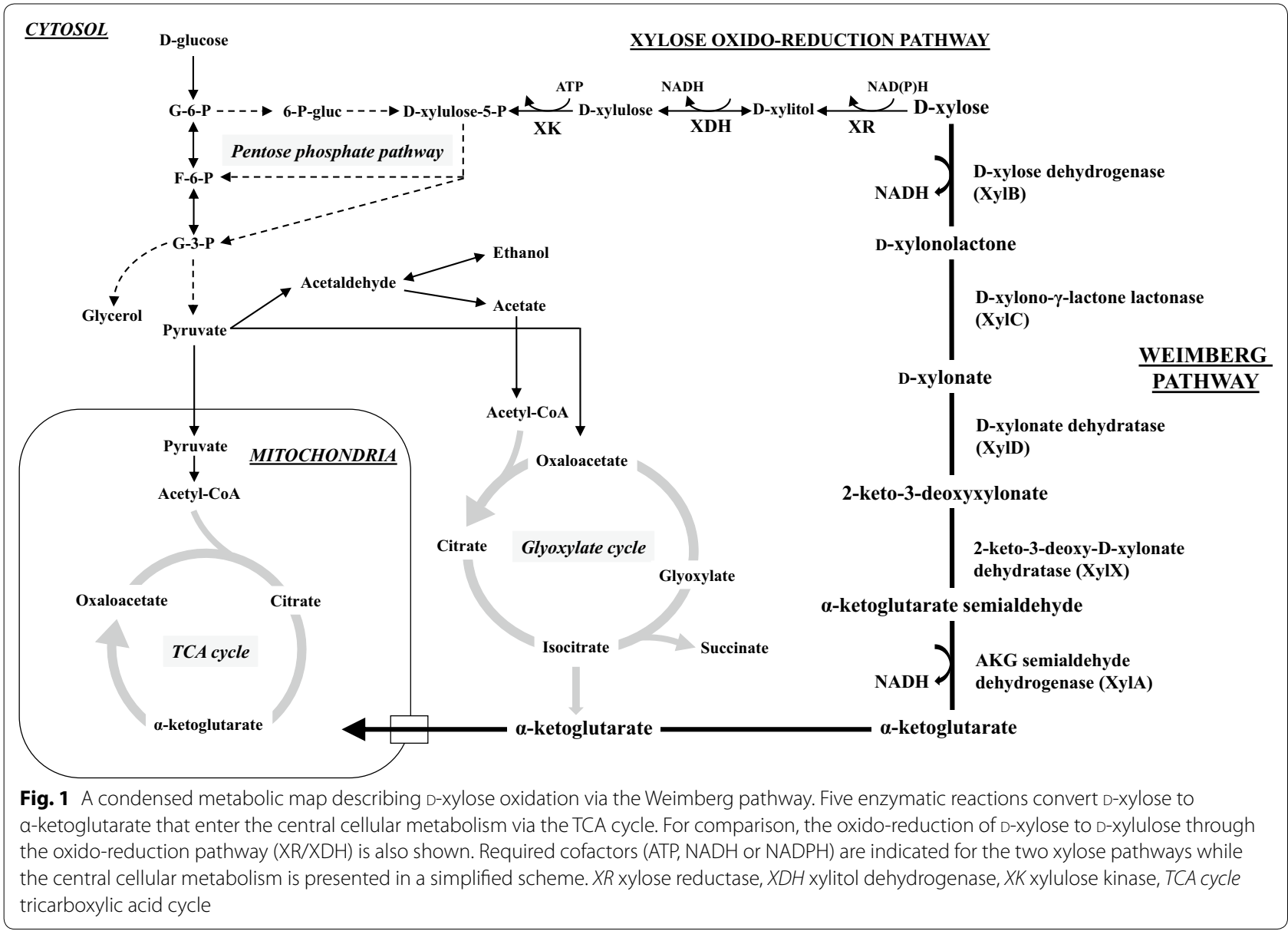


can be used as a precursor for the production of acids with novel characteristics that are not yet being produced on a large scale, such as glutaric, glutaconic and glutamic acids (Bajaj and Singhal 2011; Djurdjevic et al. 2011; Dutta et al. 2013; Fink 2013; Sailakshmi et al. 2013; Sung et al. 2005). Chemical synthesis of $\alpha$-ketoglutarate is possible through various routes, but problematic since it is a multi-step reaction partly involving toxic chemicals (Otto et al. 2011). Therefore, more attention is being directed to the biotechnological production of $\alpha$-ketoglutarate since this constitutes an environmentally friendly alternative (Aurich et al. 2012; Otto et al. 2011).

The upper part of the Weimberg pathway has already been successfully introduced in S. cerevisiae (Salusjärvi et al. 2017; Toivari et al. 2012). However, biomass formation through the Weimberg pathway can only occur if $\mathrm{D}$-xylose is fully oxidized to $\alpha$-ketoglutarate.

In the present work, genes from the complete Weimberg pathway from $C$. crescentus were introduced into $S$. cerevisiae via genetic engineering to evaluate the possibility to oxidise D-xylose, with the long-term aim to produce $\alpha$-ketoglutarate-derived bulk chemicals of industrial interest. The impact of replacing the D-xylonate dehydratase from $C$. crescentus (Cc XylD) by homologous enzymes of bacterial and archaeal origin on the yeast physiology and the functionality of the Weimberg pathway was also assessed as it has been shown that the dehydration reaction of $\mathrm{D}$-xylonate to 2-keto-3-deoxy$\mathrm{D}$-xylonate catalyzed by XylD in C. crescentus is dependent on the coordination of $\mathrm{Fe}-\mathrm{S}$ cluster(s) to three conserved cysteine residues (Andberg et al. 2016).

\section{Materials and methods}

\section{Strains, media and culture conditions}

Escherichia coli strain NEB5- $\alpha$ (New England Biolabs) was used for sub-cloning of plasmid DNA and grown at $37{ }^{\circ} \mathrm{C}$ in Luria-Bertani (LB) broth containing $5 \mathrm{~g} \mathrm{~L}^{-1}$ yeast extract, $10 \mathrm{~g} \mathrm{~L}^{-1}$ peptone, $5 \mathrm{~g} \mathrm{~L}^{-1} \mathrm{NaCl}$, pH 7.5. Bacterial transformants were selected on LB agar plates (15 $\mathrm{g} \mathrm{L}^{-1}$ agar) supplemented with $50 \mathrm{mg} \mathrm{L}^{-1}$ ampicillin for $16 \mathrm{~h}$ at $37{ }^{\circ} \mathrm{C}$. Yeast strains were grown at $30{ }^{\circ} \mathrm{C}$ and $180 \mathrm{rpm}$ in an orbital shaker under non-selective conditions in Yeast Peptone Dextrose (YPD) medium containing $10 \mathrm{~g} \mathrm{~L}^{-1}$ yeast extract, $20 \mathrm{~g} \mathrm{~L}^{-1}$ peptone and $20 \mathrm{~g}$ $\mathrm{L}^{-1} \mathrm{D}$-glucose. Competent yeast cells were prepared and transformed according to the high efficiency LiAc protocol but DMSO was added before heat shock at $42{ }^{\circ} \mathrm{C}$ for 20 min (Gietz and Schiestl 2007). Transformants generated using the CRISPR-Cas9 system (Stovicek et al. 2015) were selected on YPD media supplemented with $200 \mathrm{mg}$ $\mathrm{L}^{-1}$ geneticin and $100 \mathrm{mg} \mathrm{L}^{-1}$ nourseothricin (Jena Bioscience, Germany) to allow selection of the Cas9-kanMX and gRNA-natMX plasmids. When amdSYM was used as the selection marker, transformants were plated on synthetic media containing acetamide as the sole nitrogen source (SM-Ac) by following the protocol described by (Solis-Escalante et al. 2013). To select for cells that had recycled the amdSYM marker, cells were plated on SM containing $2.3 \mathrm{~g} \mathrm{~L}^{-1}$ fluoroacetamide (SM-FAc). Genomic S. cerevisiae DNA to be used for PCR amplification was extracted using the LiOAc/SDS lysis protocol (Lõoke et al. 2011). All restriction enzymes used where Fastdigest enzymes purchased from ThermoFisher Scientific (Massachusetts, USA). All chemicals and sugars were purchased from Sigma Aldrich (Missouri, USA).

\section{Construction of expression cassettes, targeting fragments and plasmids}

The plasmids used in this study are listed in Table 1. Primers are listed in Additional file 1: Table S1. The Weimberg pathway from $C$. crescentus was codon-optimized for S. cerevisiae and designed with promoter-terminator pairs already included, and ordered from GeneScript USA Inc. (New Jersey, USA). The five $C$. crescentus genes were delivered on four pUC57 vectors, pUC57-xylBC, pUC57-xylD, pUC57-xylX, and pUC57-xylA. The coding regions for $x y l B, x y l C, x y l D, x y l X$ and $x y l A$ were inserted in between the strong constitutive promoter-terminator pairs of the genes TEF1, TPI1, GPM1, GPD and FBA1, respectively, known to be active on D-xylose (Jin et al. 2004). pUG6-amdSYM2 harboring the two infrequent endonuclease sites $A s i \mathrm{SI}$ and $A s c \mathrm{I}$, was constructed to form a platform for long pathway fragments by amplifying a fragment with primers MCS_Cassette_f and _ $r$ using pUG6-amdSYM as template (Solis-Escalante et al. 2013). The generated fragment was digested with SacI and SalI, and AGE-purified (agarose gel electrophoresis). The template pUG6-amdSYM was digested with SacI, SalI and BbsI and simultaneously dephosphorylated with FastAP, followed by AGE-purification. The digested fragment and vector backbone was ligated, generating pUG6amdSYM2, which was fully validated by sequencing.

pAGS7 was generated by digesting the template pUG6amdSYM2 with AscI and KasI simultaneously dephosphorylated with FastAP, followed by AGE-purification. pUC57-xylBC was digested with AscI, KasI and BspHI, and AGE-purified. The resulting fragments and templates were successfully ligated, generating pAGS7, which was validated by restriction fragment analysis using $N s b \mathrm{I}$. pAGS8 was generated by digesting the template pUG6amdSYM2 with SmaI followed by AGE-purification. The pUC57-xylD was digested with RruI, FspI and AfeI; pUC57-xylX was digested with SmaI, FspI and AfeI; pUC57-xylA was digested with SmaI, FspI, and all the digested fragments were AGE-purified. The resulting fragments and template were assembled using In-Fusion 
Table 1 Plasmids used in the present study

\begin{tabular}{|c|c|c|}
\hline Plasmid name & Relevant genotype & References \\
\hline pUG-amdSYM & (A.g.)TEF1p-amdS-(A.g.)TEFt & Solis-Escalante et al. (2013) \\
\hline pUG-amdSYM2 & Ascl-MCS;(A.g.)TEF1p-amdS-(A.g.)TEFt; AsiSI & This study \\
\hline pUC57-xylBC & TEF1p-xyIB-TEF1t-TPI1p-xyIC-TPI1t & This study \\
\hline pUC57-xylD & GPM1p-xyID-GPM1t & This study \\
\hline pUC57-xylX & GPDp-xyIX-GPDt & This study \\
\hline pUC57-xylA & FBA1p-xylA-FBA1t & This study \\
\hline pUC57-xad_Hv & GPM1p-xad_Hv-GPM1t & This study \\
\hline pUC57-xad_BC & GPM1p-xyID_BC-GPM1t & This study \\
\hline pUC57-yjhG_EC & GPM1p-yjhG_Ec-GPM1t & This study \\
\hline pUC57-xylD_El & GPM1p-xyID_El-GPM1t & This study \\
\hline Cas9 & TEF1p-Cas9-CYC1t_kanMX & Stovicek et al. (2015) \\
\hline gRNA.ADE2 & SNR52p-gRNA.ADE2-SUP4t_natMX & Stovicek et al. (2015) \\
\hline pLWA25 & SNR52p-gRNA.GRE3-SUP4t_natMX & This study \\
\hline pLWA36 & SNR52p-gRNA.xyID-SUP4t_natMX & This study \\
\hline pAGS7 & pUG-amdSYM2-TEF1p-xyIB-TEF1t-TPI1p-xyIC-TPI1t & This study \\
\hline pAGS8 & pUG-amdSYM2-GPM1p-xyID-GPM1t-GPDp-xyIX-GPDt-FBA1p-xyIA-FBA1t & This study \\
\hline pAGS8H & pUG-amdSYM2-GPM1p-xad_Hv-GPM1t-GPDp-xyIX-GPDt-FBA1p-xyIA-FBA1t & This study \\
\hline pAGS8B & pUG-amdSYM2-TEF1p-xyIB-TEF1t-GPM1p-xyID-GPM1t-GPDp-xyIX-GPDt-FBA1p-xyIA-FBA1t & This study \\
\hline pAGS8HB & pUG-amdSYM2-TEF1p-xyIB-TEF1t-GPM1p-xad_Hv-GPM1t-GPDp-xyIX-GPDt-FBA1p-xyIA-FBA1t & This study \\
\hline
\end{tabular}

HD Enzyme Mix (Clontech Laboratories, California, USA) and the resulting plasmid was validated. pAGS8H was constructed by digesting the template pAGS8 with SalI and dephosphorylated with FastAP, followed by AGE-purification. pUC57-xad_Hv was digested with SalI, and the resulting, purified fragments were ligated. The generated vector was validated and correct orientation was verified with PCR using primers $\mathrm{xad}_{-} \mathrm{Hv}$ f $/$ / GPM1t_r and restriction fragment analysis using NdeI. pAGS8B and pAGS8HB were generated by cloning the TEFp-xylB-TEFt from AGS7 into pAGS8 and pAGS8H, respectively. pAGS7 was digested with $A s c \mathrm{I}$ and $A f e \mathrm{I}$ and pAGS8 and pAGS8H were digested with AscI and SmaI, and the resulting, purified fragments were ligated. The obtained vectors pAGS8B and pAGS8HB were validated by PCR and restriction fragment analysis using NdeI and AleI.

Three homologs to $C$. crescentus $x y l D$ were selected, codon-optimized for S. cerevisiae using GeneArt (ThermoFisher Scientific, Massachusetts, USA) and ordered from GeneScript USA Inc. (New Jersey, USA). The gene sequences were retrieved from the Integrative Microbial Genomes database supplied by the Joint Genome Institute (Markowitz et al. 2014).

The CRISPR-Cas9 system developed by Stovicek et al. (2015) was used for selective targeting of sites. The gRNA target sequence for GRE3 was selected using the CRISPy tool (Ronda et al. 2014) and for $x y l D$ using the E-CRISP tool (Heigwer et al. 2014). The gRNA plasmids pLWA25 and pLWA36 were constructed by amplifying the gRNA backbone as described by Stovicek et al. (2015) using a phosphorylated forward primer, 102 for pLWA25 and 263 for pLWA36, containing a tail with the specific 20-bp target sequence for GRE3 or $x y l D$, respectively, together with the phosphorylated reverse primer 103. As a template the two-micron-based replicative gRNA plasmid for targeting $A D E 2$ containing the natMX dominant marker for selection was used.

Targeting fragments for nested double homologous integration of pAGS7, pAGS8B and pAGS8HB into the GRE3 locus and pAGS8 into the intergenic region between $V A C 17$ and $M R C 1$ were constructed by PCR amplification. About $400 \mathrm{bp}$ of the $5^{\prime}$ upstream region and the $3^{\prime}$ downstream region of GRE3 were amplified from genomic $S$. cerevisiae CEN.PK 113-7D DNA using Phusion polymerase and primer pairs 84/85 (5'GRE3_AGS7), 86/87 (3'GRE3_AGS7), 84/109 (5'GRE3_AGS8) and 87/110 (3'GRE3_AGS8), generating PCR products with 50 bp homology to the flanks of $S f a \mathrm{AI} / S g s$ I cleaved plasmid AGS7 or SgsI/EheI cleaved plasmids pAGS8B and pAGS8HB. For integration of pAGS8 into the VAC17/ $M R C 1$ intergenic region, $400 \mathrm{bp}$ of the $5^{\prime}$ upstream region and the $3^{\prime}$ downstream intergenic region between VAC17 and MRC1 were amplified from genomic CEN. PK 113-7D DNA using Phusion polymerase and primer pairs 88/89 (5'VAC17_AGS8) and MRC1_f/MRC1_r ( $3^{\prime} M R C 1$ AGS8) generating PCR products with $50 \mathrm{bp}$ homology to the flanks SfaAI/SgsI cleaved plasmid 
pAGS8. For integration of pAGS7, pAGS8 and pAGS8H the amdSYM marker was included and to enable marker recycling of amdSYM a $40 \mathrm{bp}$ direct repeat with homology to the upstream region of amdSYM was included in the primers 86_3'GRE3_f_AGS7 and 3'MRC1_r_AGS8 (Solis-Escalante et al. 2013). A schematic picture of the integration of pAGS8B and pAGS8HB in the GRE3 locus using CRISPR-Cas9 is shown in Additional file 2: Figure S1.

\section{Yeast strain engineering}

The strains used and generated in this study are listed in Table 2. The upper part of the Weimberg pathway containing $x y l B$ and $x y l C$ (pAGS7) was integrated into the GRE3 locus of CEN.PK 113-7D while the lower part of the Weimberg pathway containing $x y l D, x y l X$ and $x y l A$ (pAGS8) was integrated into the VAC17/MRC1 intergenic region using nested homologous recombination. For pAGS7 integration the strain was transformed with SfaAI/SgsI-digested pAGS7 and the targeting fragments for GRE3_AGS7 described above. pAGS8 or pAGS8H were integrated by transforming the strain with SfaAI/SgsI digested pAGS8/8H and the targeting fragments for VAC17/MRC1_AGS8 described above. Transformants were selected on SM-Ac (containing acetamide as the sole nitrogen source) and correct integration was validated with primer pairs 5/87 for pAGS7 and with 5/ MRC1_r and 88/158 for pAGS8/pAGS8H. The amdSYM marker was recycled by growing the cells overnight in liquid YPD and plating a small amount of cells on SMFAc where only cells that had lost the amdSYM marker should be able to survive (Solis-Escalante et al. 2013).
Removal of the amdSYM marker was confirmed by PCR with primer pairs 155/87 for pAGS7 and 222/MRC1_r for pAGS8/pAGS8H. The resulting strains were named TMB4511 (pAGS7), TMB4515 (pAGS8), TMB4520 (pAGS7/8) and TMB4526 (pAGS7/8H).

In the second generation of strains, $x y l C$ was eliminated to allow a more gradual formation of $\mathrm{D}$-xylonate and avoid the drastic drop in intracellular $\mathrm{pH}$ as previously reported (Nygård et al. 2014). First, a control strain containing only $x y l B$ was constructed. TEFp-xylB-TEFt was amplified from pAGS7 with primers $308 / 309$ containing 50 bp flank to the GRE3 locus and transformed together with gRNA.GRE3-natMX (LWA25) into CEN. PK 113-7D already containing Cas9-kanMX. Transformants were selected on YPD supplemented with geneticin and nourseothricin and validated by amplifying parts of the integrated cassette by PCR using primers $65 / 225$. The resulting strain was named TMB4512. In a similar approach the constructed Weimberg genes present in pAGS8B and pAGS8HB was integrated into the GRE3 ORF of S. cerevisiae CEN.PK 113-7D through homologous recombination using the CRISPR-Cas9 system and a gRNA that target the GRE3 ORF as described above and in Additional file 2: Figure S1 (Stovicek et al. 2015). The yeast already expressing Cas9-kanMX was transformed with AscI and EheI digested pAGS8B and pAGS8HB plasmids, the targeting fragments for GRE3 and gRNA.GRE3-natMX (LWA25). Transformants were selected on YPD supplemented with geneticin and nourseothricin and validated by amplifying parts of the integrated cassette by PCR using primer pair $84 / 66$. The resulting strains were named TMB4530 (pAGS8B) and

Table 2 Strains used in the present study

\begin{tabular}{|c|c|c|c|}
\hline Strain name & Relevant genotype & Encoded enzymes introduced into yeast & References \\
\hline CEN.PK 113-7D & MATa URA3 HIS3 LEU2 TRP1 MAL2-8C SUC2 & - & Entian and Kötter (2007) \\
\hline TMB4545 & $\begin{array}{l}\text { VAC17/MRC1::TDH3p-GAL2 } 2^{N 376 F}-C Y C 1 t-F B A 1 p-T A L-P D C 1 t-T P 11 p- \\
\text { TKL-CPS1t, X-2::XYL-XDH-XKS1;XI-5::XYL-XDH-XKS1, XII-4::XYL- } \\
\text { XDH-XKS1 }\end{array}$ & $\mathrm{XR}, \mathrm{XDH}$ (with an optimized PPP) & Unpublished \\
\hline TMB4511 & CEN.PK 113-7D, $\triangle$ gre3::AGS7 & XylB, XylC & This study \\
\hline TMB4512 & CEN.PK 113-7D, $\triangle$ gre3::xylB & XylB & This study \\
\hline TMB4515 & CEN.PK 113-7D, vac17mrc1::AGS8 & XyID, XyIX, XylA & This study \\
\hline TMB4520 & CEN.PK 113-7D, $\triangle$ gre3::AGS7, vac17mrc1::AGS8 & XylB, XylC, XylD, XylX, XylA & This study \\
\hline TMB4526 & CEN.PK 113-7D, $\triangle$ gre3::AGS7, vac17mrc1::AGS8H & XylB, XylC, XAD_Hv, XylX, XylA & This study \\
\hline TMB4530 & CEN.PK 113-7D, $\triangle$ gre3::AGS8B & XylB, XylD, XylX, Xy|A & This study \\
\hline TMB4531 & CEN.PK 113-7D, $\triangle$ gre3::AGS8HB & XylB, XAD_Hv, XylX, XylA & This study \\
\hline TMB4569 & CEN.PK 113-7D, $\triangle$ gre3::AGS8HB, $\triangle x y|D:: x y| D \_B C$ & Xy|B, XylD_Bc, XylX, XylA & This study \\
\hline TMB4570 & CEN.PK 113-7D, $\triangle$ gre3::AGS8HB, $\triangle x y I D:: y j h G \_E C$ & XylB, YjhG_Ec, XylX, XylA & This study \\
\hline TMB4571 & CEN.PK 113-7D, sgre3::AGS8HB, $\triangle x y|D:: x y| D_{-} E l$ & XylB, XylD_El, XylX, XylA & This study \\
\hline
\end{tabular}

XR: D-xylose reductase, XDH: xylitol dehydrogenase, PPP: pentose phosphate pathway, XylB: D-xylose dehydrogenase, XyIC: Xylonolactonas, XyID: D-xylonate dehydratase, XyIX: 2-keto-3-deoxy-D-xylonate dehydratase, XylA: a-ketoglutarate semialdehyde dehydrogenase, Hv: Haloferax volcanii, Bc: Burkholderia cenocepacia, Ec: Escherichia coli, El: Ellin329 isolate 
TMB4531 (pAGS8HB). The strain TMB4530 containing pAGS8B ( $x y l B, x y l D, x y l X$ and $x y l A$ from C. crescentus) was further engineered using CRISPR-Cas9 in order to replace the D-xylonate dehydratase from $C$. crescentus with three alternative putative $x y l D$ genes from $B$. cenocepacia ( $\left.x y l D \_B c\right)$, E. coli (yjhG_Ec) and Ellin329 bacterium $\left(x y l D_{-} E l\right)$ resulting in three new strains named TMB4569, TMB4570 and TMB4571, respectively. These three strains were constructed by transforming TMB4530 containing Cas9-kanMX with either BamHI and AscI digested pUC57-xylD_Bc, pUC57-yjhG_Ec or pUC57-xylD_El together with the episomal plasmid gRNA.xylD-natMX (pLWA36). All the four $x y l D$ homologs were designed with the same promoter-terminator pairs (from GPM1) to allow homologous recombination and replacement of the $x y l D$ as shown in the schematic picture presented in Additional file 2: Figure S1 panel B. Transformants were selected on YPD supplemented with geneticin and nourseothricin and validated by amplifying the specific $x y l D$ homolog using primer pairs 269/270 (xylD_El), 271/272 (yjhG_Ec) and 273/274 $\left(x y l D \_B c\right)$.

\section{Reverse transcriptase-PCR experiments}

Strains were grown in YPD to exponential phase and cells were lysed using a bead beater (Precellys 24, Bertin Technologies, France) connected to a cooling device (Cryolys, Bertin Technologies, France) according to the supplier's instructions. RNA was extracted from the cell lysate using RNeasy Mini Kit (Qiagen, 74104) according to supplier's instructions. DNA was degraded from the RNA samples using DNA free kit from Ambion (AM1906, Life Technologies, California, USA) and incubation for $1 \mathrm{~h}$ at $37^{\circ} \mathrm{C}$. Complete removal of DNA was confirmed by PCR directly on the RNA samples using primer pair $232 / 233$ that amplify the constitutively expressed gene PFY1 encoding Profilin. RNA concentration was determined using Biodrop DUO (Biodrop Ltd, Cambridge, England). 200 ng DNA-free RNA was then used for cDNA synthesis with Superscript IV RT polymerase and oligo $\mathrm{d}(\mathrm{T})_{20}$ primer. $2.5 \mu \mathrm{M}$ oligo $\mathrm{d}(\mathrm{T})_{20}$ was mixed with $0.5 \mathrm{mM}$ dNTPs and $200 \mathrm{ng}$ RNA. The primer-RNA mix was heated to $65{ }^{\circ} \mathrm{C}$ for $5 \mathrm{~min}$. After cooling on ice SSIV buffer, $5 \mathrm{mM}$ DTT, RNAseOUT and 200 U of Superscript IV RT polymerase were added to the RNA primer mix and incubated at $50{ }^{\circ} \mathrm{C}$ for $10 \mathrm{~min}$, followed by enzyme heat inactivation for $10 \mathrm{~min}$ at $80{ }^{\circ} \mathrm{C}$. The cDNA was used for RT-PCR to verify that the Weimberg genes were transcribed in S. cerevisiae strains TMB4530, TMB4531, TMB4569, TMB4570 and TMB4571. Specific primers to amplify $x y l B, x y l D, x y l D_{-}$ $E l, y j h G_{-} E c, x y l D \_B c, x a d_{-} H v, x y l X$ and $x y l A$ are listed in Additional file 1: Table S1.

\section{In vitro enzymatic activity measurements}

Overnight cultures were inoculated in $250 \mathrm{~mL}$ shake flasks containing $25 \mathrm{~mL}$ YNB supplemented with D-glucose $\left(20 \mathrm{~g} \mathrm{~L}^{-1}\right)$ at a starting optical density of 0.25 at $620 \mathrm{~nm}$ (Ultrospec 2100 Pro spectrophotometer, Amersham Biosciences Corp., USA) and grown at $30{ }^{\circ} \mathrm{C}$ and $180 \mathrm{rpm}$. The cells were harvested at late exponential phase and the total protein was extracted using Y-PER extraction solution (Pierce, Illinois, USA) according to manufacturer's instructions. Total protein concentration was determined using Micro $\mathrm{BCA}^{\mathrm{TM}}$ Protein Assay (ThermoFisher Scientific, Massachusetts, USA) and bovine albumin standard (ThermoFisher Scientific, Massachusetts, USA) based on the assay developed by (Bradford 1976). All enzyme activities were assayed by following the rate of $\mathrm{NAD}^{+}$reduction by measuring the optical density at $340 \mathrm{~nm}\left(\mathrm{OD}_{340 \mathrm{~nm}}\right.$, Ultrospec 2100 Pro spectrophotometer, Amersham Biosciences Corp., USA). The measurements were performed in duplicates at $30{ }^{\circ} \mathrm{C}$ on a Multiskan Ascent reader (Thermo Electro Corporation, Finland) using 96-well plates and a final volume of $200 \mu \mathrm{L}$. Before adding the substrate (D-xylose or D-xylonate) to the reactions, the background formation of NADH was monitored and subtracted, if present. The standard assay mixture for all the enzymatic reactions contained $100 \mathrm{mM}$ Tris- $\mathrm{HCl} \mathrm{pH}$ 8.0, $2 \mathrm{mM} \mathrm{MgCl}_{2}$ and $2 \mathrm{mM} \mathrm{NAD}^{+}$. A concentration of $100 \mathrm{mM}$ of D-xylose was used as substrate for D-xylose dehydrogenase XylB (Toivari et al. 2012). The overall combined activity of the lower part of the pathway including XylD, XylX and XylA was assayed by following the formation of NADH using $80 \mathrm{mM}$ D-xylonate as substrate (Meijnen et al. 2009). The D-xylonate dehydratase activity was also assayed using the thiobarbituric assay (TBA) method as described by Buchanan et al. (1999) and Salusjärvi et al. (2017). $10 \mu \mathrm{L}$ of protein extract was mixed with $90 \mu \mathrm{L} 10 \mathrm{mM}$ D-xylonic acid in $10 \mathrm{mM} \mathrm{MgCl}$ and $50 \mathrm{mM}$ Tris- $\mathrm{HCl}(\mathrm{pH}$ 8.5) and incubated at $30{ }^{\circ} \mathrm{C}$ for $10 \mathrm{~min}$. After the incubation, $10 \mu \mathrm{L} 12 \%(\mathrm{w} / \mathrm{v})$ trichloroacetic acid was added and precipitated proteins were removed by centrifugation. $50 \mu \mathrm{L}$ of the supernatant was oxidized by addition of $125 \mu \mathrm{L}$ $25 \mathrm{mM}$ periodic acid/0.25 $\mathrm{M} \mathrm{H}_{2} \mathrm{SO}_{4}$ and incubated at room temperature for $20 \mathrm{~min}$. To terminate oxidation, $250 \mu \mathrm{L} 2 \%(\mathrm{w} / \mathrm{v})$ sodium (meta) arsenite in $0.5 \mathrm{M} \mathrm{HCl}$ was added. $1 \mathrm{~mL} 0.3 \%(\mathrm{w} / \mathrm{v})$ thiobarbituric acid (TBA) was added and the chromophore developed for $10 \mathrm{~min}$ at $100{ }^{\circ} \mathrm{C}$. To intensify the color, $100 \mu \mathrm{L}$ of this sample was taken and added to $100 \mu \mathrm{L}$ DMSO in a microtiter plate. The absorbance was read at $550 \mathrm{~nm}$.

\section{Dot plating}

A single-colony of each strain was grown in $5 \mathrm{~mL}$ of $\mathrm{YNB}$ supplemented with $\mathrm{D}$-glucose in a $50 \mathrm{~mL}$ conical tube 
(Sarstedt AG \& Co., Nümbrecht, Germany) and incubated at $30{ }^{\circ} \mathrm{C}$ and $180 \mathrm{rpm}$. At the end of the exponential growth phase, the cultivations were stopped and the amount of cells was normalized by dilution with $0.9 \%$ $\mathrm{NaCl}$ to an $\mathrm{OD}_{620}$ of 2.5, which corresponds to approximately $10^{7}$ colony forming units (CFU) per mL. Serial dilutions of each strain were done under sterile conditions with $0.9 \% \mathrm{NaCl}$ to obtain a coverage from $10^{5}$ to $10^{8} \mathrm{CFU} \mathrm{mL} \mathrm{mL}^{-1} .5 \mu \mathrm{L}$ of each dilution were carefully dot plated in YNB-agar plates supplemented with D-glucose $\left(5 \mathrm{~g} \mathrm{~L}^{-1}\right)$, D-xylose $\left(20 \mathrm{~g} \mathrm{~L}^{-1}\right)$ or a mixture of D-glucose/Dxylose (5 and $20 \mathrm{~g} \mathrm{~L}^{-1}$, respectively) and incubated at $30{ }^{\circ} \mathrm{C}$ for $48 \mathrm{~h}$.

\section{Aerobic cultivations in bioreactor}

Bioreactor experiments were performed in lab-scale $3 \mathrm{~L}$ reactors Braun Biostat CT (B. Braun International, Germany) or Sartorius Biostat A+ (Sartorius, Germany) with an initial working volume of $1 \mathrm{~L}$. Temperature was $30{ }^{\circ} \mathrm{C}$, stirring $500 \mathrm{rpm}$ using dual six-bladed Rushton turbines and sparging was $0.5 \mathrm{vvm}$ air. As in the case of shake-flask cultivations, the medium also contained $6.7 \mathrm{~g}$ $\mathrm{L}^{-1} \mathrm{YNB}$ with ammonium sulfate. For the batch experiments, the initial carbon source was $5 \mathrm{~g} \mathrm{~L}^{-1} \mathrm{D}$-glucose. When D-glucose was depleted, $20 \mathrm{~g}$ of D-xylose was added by injecting $50 \mathrm{~mL}$ of a $400 \mathrm{~g} \mathrm{~L}^{-1} \mathrm{D}$-xylose solution. $\mathrm{pH}$ was set to 5.5 and controlled by the addition of $5 \mathrm{M} \mathrm{KOH}$. In the case of the fed-batch experiments, the initial substrate increased to $10 \mathrm{~g} \mathrm{~L}^{-1} \mathrm{D}$-glucose. D-Xylose was subsequently added to a concentration of $10 \mathrm{~g} \mathrm{~L}^{-1}$, by pulsing $50 \mathrm{~mL}$ of a $200 \mathrm{~g} \mathrm{~L}^{-1} \mathrm{D}$-xylose solution. During fed-batch mode a $40 \mathrm{~g} \mathrm{~L}^{-1} \mathrm{D}$-glucose solution was fed at a rate of $0.2 \mathrm{~g} \mathrm{D}$-glucose $\mathrm{h}^{-1}$.

\section{Aerobic cultivations in shake-flasks}

Prior to the aerobic cultivation, yeast pre-cultures were obtained by transferring a loop of cells from the glycerol stocks into $5.0 \mathrm{~mL}$ of YNB defined medium $\left(6.7 \mathrm{~g} \mathrm{~L}^{-1}\right.$ Yeast Nitrogen Base without amino acids (Becton, Dickinson and Company, USA) supplemented with $20 \mathrm{~g} \mathrm{~L}^{-1}$ D-glucose. The $\mathrm{pH}$ of the medium was adjusted to 6.0 by the addition of $\mathrm{H}_{2} \mathrm{SO}_{4}$ or $\mathrm{KOH}$. Cells were grown in a $50 \mathrm{~mL}$ sterile conical tube and incubated at $30{ }^{\circ} \mathrm{C}$ and $180 \mathrm{rpm}$ until the end of the exponential growth phase was reached. At that moment, cells corresponding to an $\mathrm{OD}_{620 \mathrm{~nm}}$ of 0.1 were transferred to $100 \mathrm{~mL}$ YNB D-glucose in $1000 \mathrm{~mL}$ baffled shake-flasks and incubated at $30{ }^{\circ} \mathrm{C}$ and $180 \mathrm{rpm}$. This intermediate step was used for cell proliferation in order to start the aerobic cultivations with high levels of biomass. At the end of the exponential phase, an amount of cells corresponding to an $\mathrm{OD}_{620 \mathrm{~nm}}$ of 14 was harvested, centrifuged ( $5 \mathrm{~min}, 4000 \mathrm{rpm}$ ) and washed once by resuspension in $0.9 \% \mathrm{NaCl}$. Aerobic cultivations were then conducted in $250 \mathrm{~mL}$ baffled shakeflasks containing $25 \mathrm{~mL}$ of YNB defined media buffered to $\mathrm{pH} 6.0$ and supplemented with $5 \mathrm{~g} \mathrm{~L}^{-1}$ D-xylose. After the inoculum, the shake-flasks were incubated at $30{ }^{\circ} \mathrm{C}$ and $180 \mathrm{rpm}$ for $100 \mathrm{~h} . \mathrm{OD}_{620 \mathrm{~nm}}$ was monitored over time and samples were taken for metabolite analysis using HPLC (cf. below). The final $\mathrm{pH}$ was measured at the end of the cultivations.

\section{Metabolite analysis and cell dry weight determination}

The measurement of the extracellular concentrations of D-xylose and D-xylonate was done by ultra-performance liquid chromatography (UPLC, Waters, Massachusetts, USA) through a novel method specifically developed for the analysis of D-xylonate (Almqvist et al. 2017). Calibration was made with a standard solution containing $\alpha$-ketoglutarate, D-xylose, D-xylitol, D-xylonate and D-glucose. Prior to analysis, samples were centrifuged (2 min, 13,000 rpm), filtered $(0.2 \mu \mathrm{m})$ and diluted with ultra-pure $\mathrm{H}_{2} \mathrm{O}$ if necessary. D-Xylose concentrations were also measured using a Waters HPLC system equipped with RI-detector $\left(45^{\circ} \mathrm{C}\right.$ cell temperature). The column was a $300 \times 7.8 \mathrm{~mm}, 9 \mu \mathrm{m}$ Aminex HPX-87P column (BIO-RAD, Hercules, CA, USA). The column temperature was $85^{\circ} \mathrm{C}$, the mobile phase $\left(0.6 \mathrm{~mL} \mathrm{~min}{ }^{-1}\right)$ consisted of ultra-pure water (18.2 $\mathrm{M} \Omega$ ) from an ELGA Purelab Flex unit (Veolia Water, Paris, France). Metabolites were analysed using a Waters HPLC system equipped with RI-detector $\left(45^{\circ} \mathrm{C}\right.$ cell temperature). The column was a $300 \times 7.8 \mathrm{~mm}, 9 \mu \mathrm{m}$ Aminex HPX- $87 \mathrm{H}$ column (BIO-RAD, Hercules, CA, USA). The column temperature was $60^{\circ} \mathrm{C}$, the mobile phase $\left(0.6 \mathrm{~mL} \mathrm{~min}^{-1}\right)$ consisted of $5 \mathrm{mM} \mathrm{H}_{2} \mathrm{SO}_{4}$ in ultra-pure water. Calibration was made with a standard solution containing $\alpha$-ketoglutarate, D-xylose, D-xylitol, acetate, ethanol and glycerol. D-Xylonate concentrations were also measured using the hydroxamate method (Lien 1959) as described by Toivari et al. (2010). The D-xylonate concentration in the samples was determined by comparison with a standard curve using lithium xylonate from SigmaAldrich. The cell dry weight (CDW) was obtained from optical density $\left(\mathrm{OD}_{620}\right)$ through a correlation curve. CDW was initially determined in triplicate by centrifuging samples at 13,000 rpm for $5 \mathrm{~min}$. The cells were washed by resuspension in ultra-pure water followed by centrifugation. The washed cells were then resuspended in ultra-pure water and transferred to pre-dried and pre-weighed $5 \mathrm{~mL}$ glass test tubes. The test tubes were dried in a convection oven (Termaks, Norway) at $105{ }^{\circ} \mathrm{C}$ for $16-24 \mathrm{~h}$ and then placed in a desiccator until equilibration to room temperature. After this analysis, it 
was determined that an $\mathrm{OD}_{620}$ of 1.0 corresponded to a CDW of $0.40 \mathrm{~g} \mathrm{~L}^{-1}$.

\section{Primary nucleotide accession numbers}

The primary nucleotide accession numbers of the genes of bacterial/archaeal origin heterologously expressed in S. cerevisiae were obtained from GenBank or the Integrative Microbial Genome database (IMG) and are listed in Table 3. These Weimberg pathway genes codonoptimized towards S. cerevisiae was deposited in GenBank with the accession numbers listed in Table 3. The percent sequence identity with the $C$. crescentus XylD is presented for the four XylD homologs used and was obtained by aligning the protein sequences using the multiple sequence alignments tool Clustal Omega developed at the European Bioinformatics Institute (EMBLEBI) (McWilliam et al. 2013).

\section{Results}

Expression of the Weimberg pathway from C. crescentus for D-xylose assimilation in S. cerevisiae

Several different $S$. cerevisiae strains were constructed with varying combinations of the Weimberg pathway genes from $C$. crescentus integrated in the genome. In strain, TMB4511, only the first two genes, $x y l B$ and $x y l C$, were integrated in the $S$. cerevisiae genome while in another strain, TMB4520, the entire Weimberg pathway genes were integrated $(x y l B, x y l C, x y l D, x y l X$ and $x y l A)$. To prevent D-xylitol formation, GRE3 encoding a native aldose reductase was deleted in all the $S$. cerevisiae strains carrying the Weimberg pathway. The growth capacity of the strains was evaluated by dot plating on media containing $\mathrm{D}$-glucose, $\mathrm{D}$-xylose or a mixture of both carbon sources (Fig. 2). When D-glucose was the sole carbon source, all strains grew, which was

Table 3 Nucleotide accession numbers of the bacterial/archeal genes used in the study

\begin{tabular}{|c|c|c|c|c|c|}
\hline \multirow[t]{2}{*}{ Gene name } & \multirow[t]{2}{*}{ Organism } & \multicolumn{3}{|c|}{ Accession number } & \multirow[t]{2}{*}{ \% seq. identity with Cc XyID } \\
\hline & & GenBank & IMG & GenBank codon-opt ${ }^{b}$ & \\
\hline D-Xylose dehydrogenase $x y / B$ & C. crescentus & YP_002516237.1 & & MG681087 & \\
\hline D-Xylonolactonase $x y / C$ & C. crescentus & YP_002516236.1 & & MG681088 & \\
\hline D-Xylonate dehydratase $x y / D$ & C. crescentus & YP_002516235 & & MG681089 & 100 \\
\hline 2KD-dehydratase $x y I X$ & C. crescentus & YP_002516239.1 & & MG681090 & \\
\hline $\begin{array}{l}\text { AKG semialdehyde dehydrogenase } \\
x y / A\end{array}$ & C. crescentus & YP_002516238.1 & & MG681091 & \\
\hline D-Xylonate dehydratase yjhG & E. coli & WP_000116326.1 & & MG681092 & 29.4 \\
\hline D-Xylonate dehydratase xad & H. volcanii & WP_004041116.1 & & MG681093 & 18.6 \\
\hline D-Xylonate dehydratase & Ellin329 bact ${ }^{\mathrm{a}}$ & & 2626539782 & MG681094 & 73.9 \\
\hline Dihydroxy-acid dehydratase & B. cenocepacia H111 & & 2533426636 & MG681095 & 62.7 \\
\hline
\end{tabular}

a Full name: Ellin329 bacterium JGI 000192CP-E08

b GenBank accession number for genes codon optimized for Saccharomyces cerevisiae

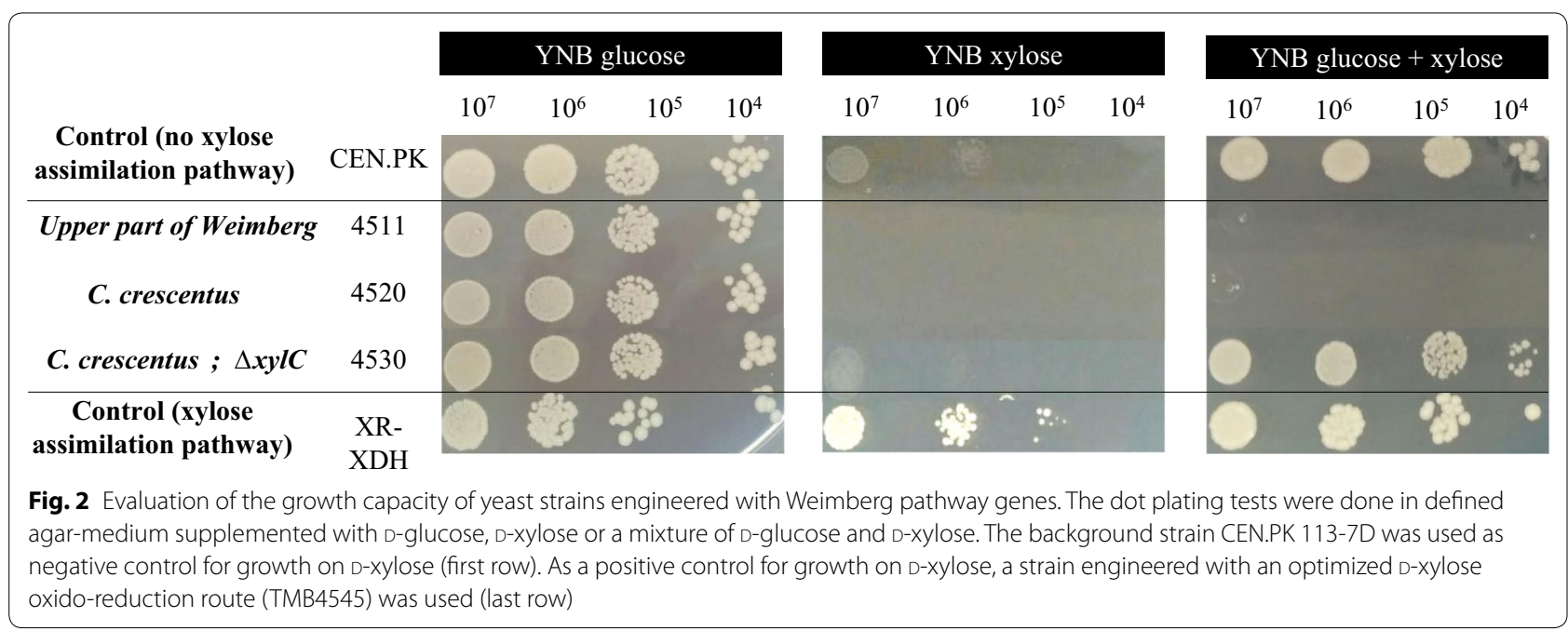


expected since the D-glucose metabolism is not directly connected with the $\mathrm{D}$-xylose assimilation through the Weimberg pathway. However, when D-glucose was used in combination with D-xylose as carbon source, growth of strains carrying part or all Weimberg pathway genes (TMB4511 and TMB4520, respectively) was inhibited, indicating that D-xylose conversion, either in part or fully, through the Weimberg pathway was inhibitory to $S$. cerevisiae. This is in line with previous published results by Nygård and coworkers in 2014 who showed that the expression of the D-xylono- $\gamma$-lactone lactonase encoding gene $x y l C$ led to a rapid conversion of $\mathrm{D}$-xylono- $\gamma$ lactone to $\mathrm{D}$-xylonate, decreasing the intracellular $\mathrm{pH}$ and, consequently, initiating cell death. A more gradual D-xylonate formation was obtained by solely expressing the $\mathrm{D}$-xylose dehydrogenase encoding gene $x y l B$ since the conversion of D-xylono- $\gamma$-lactone into D-xylonate also occurs spontaneously at neutral $\mathrm{pH}$ (Buchert and Viikari 1988; Nygård et al. 2014). In the present study, a strain was therefore constructed with the full Weimberg pathway from $C$. crescentus except for $x y l C$ integrated in the genome (Additional file 2: Figure S1). In this way, it was possible to evaluate whether the absence of the D-xylono$\gamma$-lactone lactonase activity would also be beneficial when the complete Weimberg pathway was expressed in S. cerevisiae. The resulting strain, TMB4530, was indeed able to grow on D-glucose also in the presence of D-xylose in contrast to the strains containing xylC (TMB4511, TMB4520). However, TMB4530 was still not able to grow on D-xylose as the sole carbon source (Fig. 2).
Expression of the Weimberg pathway genes from $C$. crescentus in S. cerevisiae enables bioconversion of D-xylose to D-xylonate via D-xylose dehydrogenase

Strain TMB4530, containing $x y l B, x y l D, x y l X$ and $x y l A$, was evaluated by bioreactor experiments in two steps: initial cell growth on D-glucose in a batch phase followed by a pulse addition of $\mathrm{D}$-xylose to allow bioconversion at high cell density. In the first $24 \mathrm{~h}$ of the batch experiments, cells were propagated in YNB supplemented with D-glucose. After depletion of the D-glucose and the formed ethanol and acetate, the bioconversion phase was initiated by the addition of D-xylose. During this period, the specific $\mathrm{D}$-xylose uptake rate $(0.027 \pm 0.002 \mathrm{cmol}$ $\mathrm{D}$-xylose cmol biomass ${ }^{-1} \mathrm{~h}^{-1}$ ) matched the $\mathrm{D}$-xylonate production rate $(0.024 \pm 0.001 \mathrm{cmol} \mathrm{D}$-xylonate $\mathrm{cmol}$ biomass $^{-1} \mathrm{~h}^{-1}$ ) (data not shown). Cell growth could not be sustained by $\mathrm{D}$-xylose during the bioconversion phase; instead biomass was estimated to decrease at a rate of $0.006 \pm 0.001 \mathrm{~h}^{-1}$. In order to maintain cell viability, a fed-batch experiment was designed where D-glucose was fed during the bioconversion phase. The fed-batch experiment consisted of three phases (Fig. 3): initial growth phase on D-glucose followed by a pulse of D-xylose, where non-growing cells assimilated the pentose for $24 \mathrm{~h}$. Finally, the third phase consisted of a prolonged fed-batch phase where D-glucose was fed at a slow rate of $0.2 \mathrm{~g} \mathrm{~h}^{-1}$. This set-up allowed the comparison of the D-xylose uptake efficiency by both growing and non-growing cells in the same experiment. The measurements showed that D-xylose uptake rate was not affected

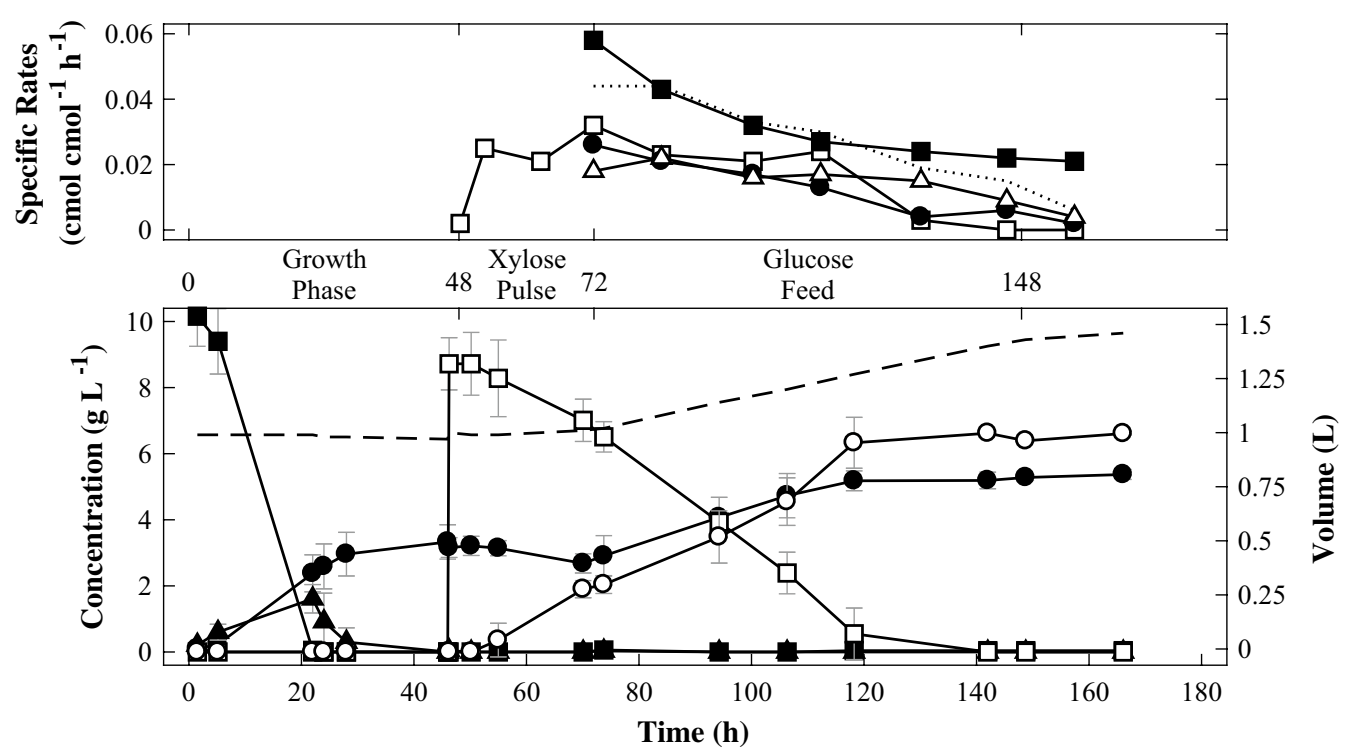

Fig. 3 Fed-batch experiments with strain TMB4530 ( $x y \mid B, x y I D, x y I X, x y / A)$. D-Glucose (filled square), biomass (filled circle), ethanol (filled triangle), D-xylose (square), D-xylonate (circle), $\mathrm{CO}_{2}$ (triangle) and liquid volume (straight line). The dotted line represents the sum of specific productivity of biomass and $\mathrm{CO}_{2}$. All experiments were performed in biological duplicates and standard deviations are indicated with error bars 
by $\mathrm{D}$-glucose feeding. In the growth phase, D-glucose depletion was reached with TMB4530 after approximately $20 \mathrm{~h}$ of cultivation. After $46 \mathrm{~h}$, a pulse of D-xylose was added and the pentose was taken up at a specific rate of $0.025 \pm 0.004 \mathrm{cmol} \mathrm{D}$-xylose $\mathrm{cmol}^{\text {biomass }}{ }^{-1} \mathrm{~h}^{-1}$ independent of the D-xylose concentration (Fig. 3). The assimilated D-xylose was converted to D-xylonate at a conversion rate of $0.020 \pm 0.005 \mathrm{cmol}_{\text {biomass }}{ }^{-1} \mathrm{~h}^{-1}$, reaching full conversion after $140 \mathrm{~h}$. No other products could be detected from D-xylose.

\section{Expression of $x y I D$ from $H$. volcanii does not convey D-xylonate conversion}

Heterologous expression of the C. crescentus Weimberg pathway in $S$. cerevisiae enables bioconversion of $\mathrm{D}$-xylose to $\mathrm{D}$-xylonate, however, the $\mathrm{D}$-xylonate is apparently not converted further as no downstream products can be detected. The dehydration reaction converting D-xylonate to 2-keto-3-deoxy-D-xylonate is catalysed by a dehydratase (XylD) belonging to the IlvD/EDD protein family that requires an iron-sulfur cluster $(\mathrm{Fe}-\mathrm{S})$ for its catalytic activity. Iron-sulfur cluster containing proteins of bacterial origin are known to be challenging to express in eukaryotes due to differences in the complicated cell machinery responsible for the loading of the Fe-S cluster onto the apoprotein (Benisch and Boles 2014). As an alternative to iron-sulphur cluster requiring Cc XylD, there are $\mathrm{D}$-xylonate dehydratases found in Archaea that belong to the enolase family and require metal ions (such as $\mathrm{Mg}^{2+}$ ) for its function rather than
Fe-S clusters (Johnsen et al. 2009; Petsko and Ringe 2004; Faller et al. 1977). Therefore, the C. crescentus xylD in strain TMB4530 containing $x y l B, x y l D, x y l X$ and $x y l A$ was replaced with the counterpart from the halophilic archaeon $H$. volcanii (HVO_B0038A, xad_Hv), generating strain TMB4531. The same three phase-set up as previously described for TMB4530 was used to test D-xylose conversion with a low simultaneous D-glucose co-consumption. In the first batch phase $(24 \mathrm{~h})$ cells were propagated in YNB supplemented with D-glucose to high cell density and then the second phase was initiated after $30 \mathrm{~h}$ by adding a pulse of $\mathrm{D}$-xylose to allow bioconversion by the non-growing cells produced in the first phase. The third phase was a prolonged fed-batch phase where D-glucose was added at a slow rate of $0.2 \mathrm{~g} \mathrm{~h}^{-1}$ to maintain cell viability during the $\mathrm{D}$-xylose bioconversion. TMB4531 reached a maximum uptake rate $(0.06 \mathrm{cmol}$ D-xylose cmol biomass ${ }^{-1} \mathrm{~h}^{-1}$ ) when the highest concentration of $\mathrm{D}$-xylose was present, declining after that timepoint. During the initial part of the fed-batch phase, the specific biomass and $\mathrm{CO}_{2}$ productivity were identical to TMB4530. In contrast, the carbon flux appeared to be shifted towards $\mathrm{CO}_{2}$ formation at later stages (Figs. 3, 4). The decrease in biomass yield correlated well with the D-xylonate concentrations, suggesting increased maintenance requirements due to the need of secreting the acid to maintain the intracellular $\mathrm{pH}$, as previously suggested (Nygård et al. 2014). In both TMB4530 and TMB4531 the sum of biomass and $\mathrm{CO}_{2}$ production rates matched the D-glucose uptake rate (dotted lines in Figs. 3, 4), which

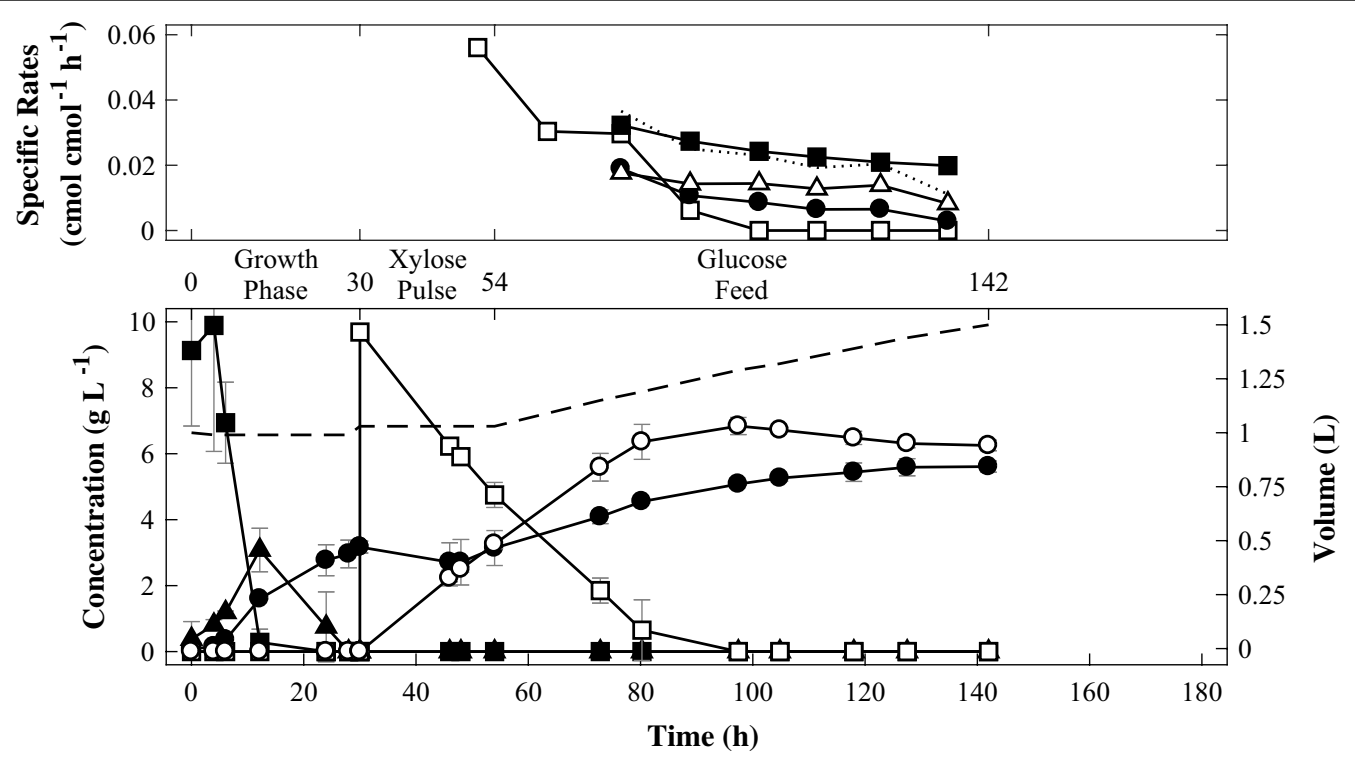

Fig. 4 Fed-batch experiments with strain TMB4531 ( $x y \mid B$, xad_HV, $x y|X, x y| A)$. D-Glucose (filled square), biomass (filled circle), ethanol (filled triangle), $\mathrm{D}$-xylose (square), D-xylonate (circle), $\mathrm{CO}_{2}$ (triangle) and liquid volume (straight line). The dotted line represents the sum of specific productivity of biomass and $\mathrm{CO}_{2}$. All experiments were performed in biological duplicates and standard deviations are indicated with error bars 
suggested that D-glucose was converted into biomass and $\mathrm{CO}_{2}$ while D-xylose was quantitatively converted into $\mathrm{D}$-xylonate (note that the concentrations decreased due to dilution during the fed-batch).

The increased D-xylose bioconversion rate in TMB4531 compared to TMB4530 could indicate that $H$. volcanii XylD ( $H v$ XAD) was able to convert some of the D-xylonate to 2-keto-3-deoxy-D-xylonate, thereby increasing the intracellular $\mathrm{pH}$ and the fitness of the cells. 2-keto-3-deoxy-D-xylonate was not included in the standard solution used in the UHPLC and HPLC analyses since it is not commercially available. But as shown in Additional file 3: Figure S2, no unidentified peaks were seen in the chromatograms from the bioreactor experiments. Proteomics analysis of TMB4531 in a parallel study indicated that $H v$ XAD was not produced in TMB4531, while $C c$ XylD was present in TMB4530 (data not shown). Synthesis of the Fe-S Cc XylD in TMB4530 might increase the metabolic burden in this strain, which would explain the lower $\mathrm{D}$-xylose bioconversion rate in TMB4530 in comparison to TMB4531 that lacks the $\mathrm{Fe}-\mathrm{S}$ XylD protein.

\section{Evaluation of three XyID homologs from the IlvD/EDD family to improve $D$-xylonate conversion}

Although Cc XylD was produced in TMB4530, the functionality of this protein remained unclear. In an attempt to improve D-xylonate conversion, we evaluated three XylD homologs of the IlvD/EDD family originating from B. cenocepacia, E. coli and an unknown organism denoted as Ellin329 bacterium, for their functionality in yeast by integration into TMB4530 replacing $x y l D$, and resulting in strains TMB4569, TMB4570 and TMB4571, respectively (Additional file 2: Figure S1). These XylD homologs were chosen based on the difference in protein sequence identity as compared to $C$. crescentus XylD (Table 3 ) and the fact that they had previously not been characterized in S. cerevisiae.

Initial evaluation showed that all strains grew well on minimal medium supplemented with D-glucose, but no growth could be observed when $\mathrm{D}$-xylose was used as the sole carbon source (data not shown). Subsequently, the biocatalytic capacities of the strains to convert D-xylose into D-xylonate and downstream products were assessed. For these tests, cells were first grown to exponential phase in medium containing D-glucose. After harvest, about $5 \mathrm{~g} \mathrm{~L}^{-1}$ biomass was transferred to a mineral medium supplemented with a small amount of D-xylose $\left(5 \mathrm{~g} \mathrm{~L}^{-1}\right)$ and aerobic cultivations were initiated in shake flasks (Fig. 5). For these experiments two control strains containing only part of the pathway were also evaluated under the same conditions namely TMB4511 containing $x y l B$ and $x y l C$ and TMB4512 containing only $x y l B$.
The results showed that both control strains TMB4511 and TMB4512 (i.e. the strain producing both XylB and $\mathrm{XylC}$ and the strain producing $\mathrm{XylB}$, respectively) converted $\mathrm{D}$-xylose to $\mathrm{D}$-xylono- $\gamma$-lactone/D-xylonate at higher rates than all the strains containing genes from the lower pathway. This suggested that the lower part of the pathway put some metabolic burden on these strains since no downstream products from D-xylonate could be detected in the current set up. In strain TMB4511, rapid $\mathrm{D}$-xylonate production was expected since the strain can enzymatically convert $\mathrm{D}$-xylono- $\gamma$-lactone to $\mathrm{D}$-xylonate; however this was also observed for the control TMB4512 that lacks $\mathrm{XylC}$ and has to rely on the spontaneous opening of the lactone ring to form $\mathrm{D}$-xylonate. This can be explained by the analytical method used, called the hydroxamate method that in the current setup does not distinguish between the lactone and the acid form since all the D-xylonate is converted to D-xylono- $\gamma$-lactone and then measured colorimetrically.

\section{Investigation of non-functional steps for yeast strains carrying the whole Weimberg pathway}

As D-xylonate was the main product in all S. cerevisiae strains carrying the Weimberg pathway, we verified that each strain expressed the correct heterologous genes. Reverse transcriptase PCR was carried out on cDNA derived from exponentially growing cells in D-glucose (D-xylose was not added since the expression in yeast is constitutive and not regulated by D-xylose as in C. crescentus). The results confirmed that all the engineered $S$. cerevisiae strains expressed $x y l B, x y l X$ and $x y l A$ and each strain expressed one unique $x y l D$ homolog: TMB4530$x y l D$ from $C$. crescentus, TMB4569-xylD from B. cenocepacia, TMB4570-yjhG from E. coli, TMB4571-xylD from Ellin329 isolate, but also TMB4531 carrying $x a d$ from $H$. volcanii (Fig. 6). Due to the absence of XylD protein in the strain carrying $H$. volcanii, the other strains were also tested and the presence of the corresponding proteins was confirmed (data not shown).

As a third step, the activity of the Weimberg enzymes was assayed. The D-xylose dehydrogenase (XylB) activity was determined enzymatically in cell extract by monitoring the NADH formation at $340 \mathrm{~nm}$ and the results showed an activity of $0.2-0.3 \mu \mathrm{mol} \mathrm{min}{ }^{-1} \mathrm{mg}^{-1}$ in the different strains (Table 4). The activity of the lower part of the pathway including $\mathrm{D}$-xylonate dehydratase (XylD), 2-keto-3-deoxy-D-xylonate dehydratase (XylX) and the $\alpha$-ketoglutarate semialdehyde dehydrogenase (XylA) was assessed through the combined activity of the enzymes by following the formation of NADH from D-xylonate. However, no enzymatic activity could be detected (data not shown). In order to validate both oxidation assays, protein extract from $\mathrm{D}$-xylose-grown $C$. crescentus 


\section{Replicate 1}
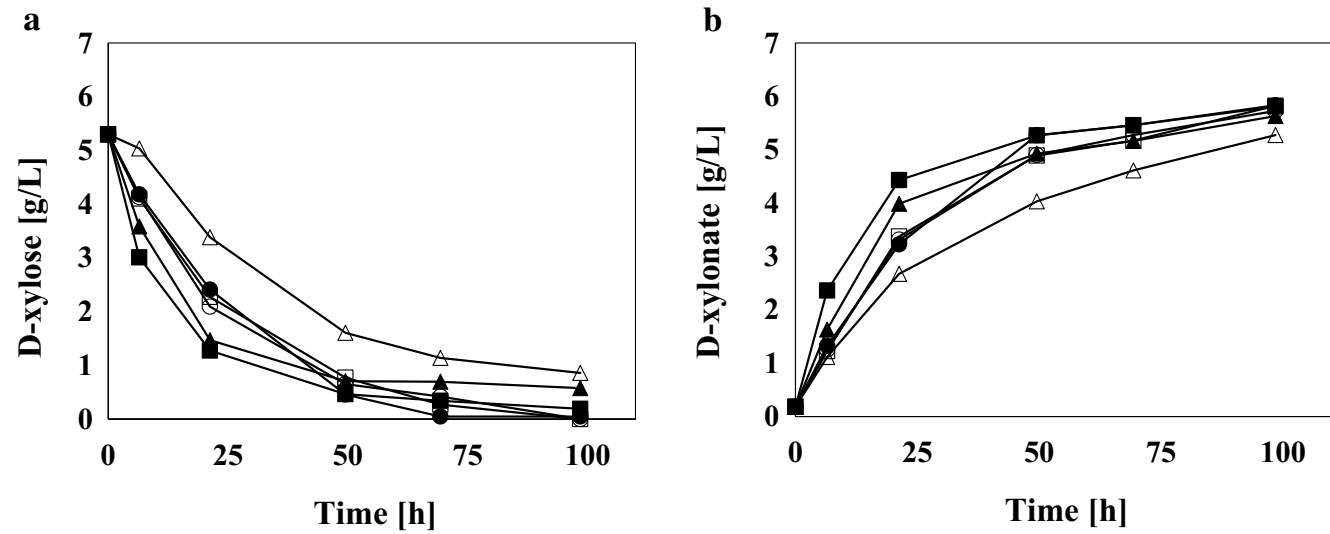

\section{Replicate 2}
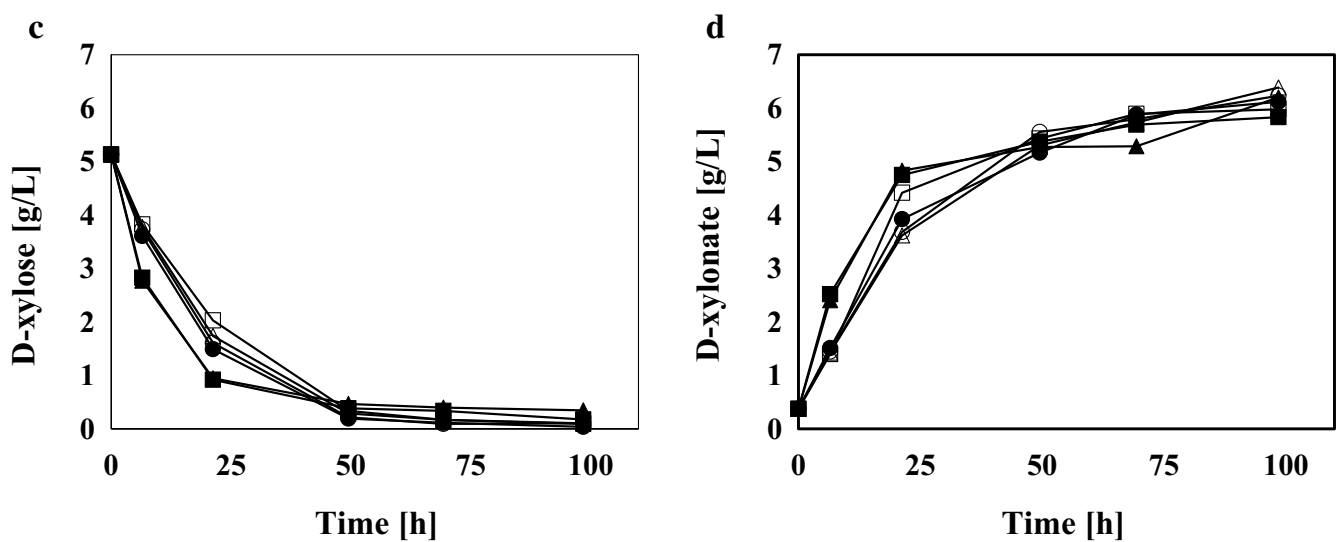

Fig. 5 Aerobic bioconversion of D-xylose into D-xylonate in recombinant Saccharomyces cerevisiae strains. TMB4511 (xylB, xy/C: filled square), TMB4512 ( $x y \mid B$ : filled triangle), TMB4530 ( $x y|B, x y| D, x y|X, x y| A$ : filled circle), TMB4569 ( $x y|B, x y| D \_B C, x y|X, x y| A$ : square), TMB4570 ( $x y\left|B, y j h G \_E C, x y\right| X$,

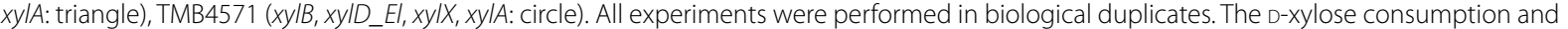
D-xylonate formation in replicate 1 is shown in the upper $\mathbf{a}$ and $\mathbf{b}$, respectively. The lower $\mathbf{c}$ and $\mathbf{d}$ show D-xylose consumption and D-xylonate formation in replicate 2 , respectively

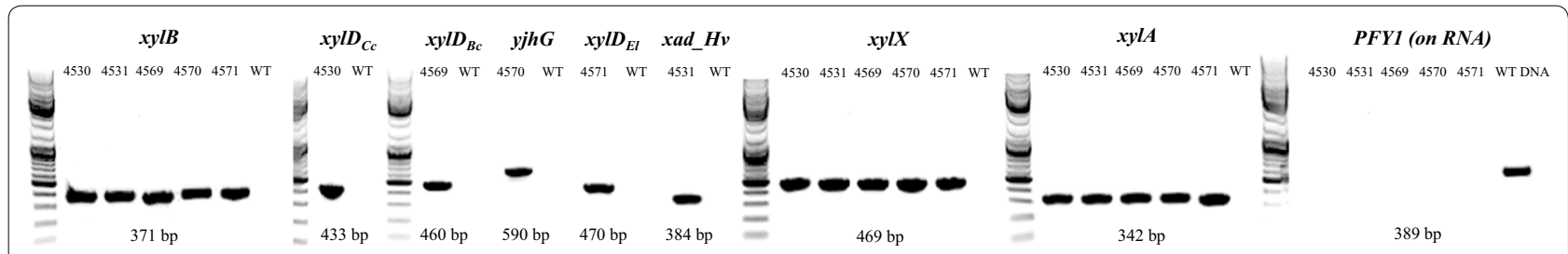

Fig. 6 Reverse-transcriptase PCR analysis to verify gene expression of Weimberg pathway genes. RNA from five different strains exponentially growing on D-glucose was purified and used for CDNA synthesis. Reverse-transcriptase PCR was performed on the CDNA using primers specific for each Weimberg pathway gene. Amplification of $x y|B, x y| A$ and $x y \mid X$ was carried out in all strains while different $x y \mid D s$ was amplified in each individual strain as follows: xyID from Caulobacter crescentus in TMB4530, xad from Haloferax volcanii in TMB4531, xyID from Burkholderia cenocepacia in TMB4569, yjhG from Escherichia coli in TMB4570 and xyID from Ellin329 isolate in TMB4571. CEN.PK 113-7D (WT) was used as negative control. Before CDNA synthesis the RNA purity (i.e. removal of DNA) was examined using a control PCR that amplifies the constitutively expressed gene PFY1 encoding Profilin 
Table 4 Specific activity of D-xylose dehydrogenase, XylB, in different recombinant $S$. cerevisiae strains

\begin{tabular}{lll}
\hline Strain & Spec. activity $\left(\boldsymbol{\mu} \mathbf{m o l} \mathbf{~ m i n}^{\mathbf{- 1}} \mathbf{~ m g}^{-\mathbf{1}}\right)$ & STDEV \\
\hline TMB4511 & 0.21 & \pm 0.04 \\
TMB4530 & 0.23 & \pm 0.05 \\
TMB4569 & 0.31 & \pm 0.04 \\
TMB4570 & 0.24 & \pm 0.03 \\
TMB4571 & 0.31 & \pm 0.04 \\
TMB4515 & 0.00 & \pm 0.03 \\
\hline
\end{tabular}

The enzymatic activity of XyIB that catalyzes the conversion of D-xylose into D-xylono- $\gamma$-lactone was assayed in five strains including: TMB4511 (XylB, XylC), TMB4530 (XylB, CC XyID, XyIX, XyIA), TMB4569 (XylB, BC XyID, xyIX, xylA), TMB4570 (XylB, Ec YjhG_Ec, XylX, XylA) and TMB4571 (XyIB, El XyID_El, xylX, xylA). As a control strain, TMB4515 that contains only the lower part of the pathway including CC XyID, XyIX and XyIA was used

cells was used as positive control and it showed enzymatic activity both for XylB and for the three enzymes XylD/XylX/XylA using D-xylose and D-xylonate as substrate, respectively, which confirmed the robustness of the assays. D-Glucose-grown C. crescentus showed no activity of the tested enzymes (unpublished data). To specifically assay the D-xylonate dehydratase activity, the thiobarbituric acid (TBA) method was also used (Buchanan et al. 1999; Salusjärvi et al. 2017). No activity could be detected, indicating that none of the XylD homologous were active in vitro (data no shown). This is in line with the recent report from Salusjärvi et al. (2017) where no XylD activity was detected unless FRA2 gene was deleted.

These results suggest that although the genes encoding the Weimberg pathway could be expressed and transcribed in S. cerevisiae, the functionality of the three proteins converting $\mathrm{D}$-xylonate into $\alpha$-ketoglutarate remained unknown. If the D-xylonate is converted further it is below detection limit in our experimental setup. Proteomics data could confirm that all the bacterial $\mathrm{Fe}-\mathrm{S}$ XylDs were present in the correct strain, yet the assembly of the $\mathrm{Fe}-\mathrm{S}$ cluster onto the apoprotein could be the limiting factor for producing an active protein.

\section{Discussion}

In the present study, the expression of the genes encoding for the entire Weimberg pathway in the eukaryote S. cerevisiae had the objective to provide an alternative entry point for D-xylose into the yeast central carbon metabolism with possibilities to produce new compounds that are intermediates or derivatives of the TCA cycle. The $C$. crescentus Weimberg pathway was chosen since the corresponding genes had already been integrated and proven to be functionally expressed in the bacteria C. glutamicum and P. putida (Meijnen et al. 2009; Radek et al. 2014). In S. cerevisiae, the upper part of the pathway including $x y l B$ and $x y l C$ from $C$. crescentus has been successfully expressed, enabling D-xylonate production from D-xylose (Nygård et al. 2014). In a recent study of the Dahms pathway in S. cerevisiae it was also shown that deletion of FRA2 encoding a repressor of iron regulon transcription resulted in some activity of the D-xylonate dehydratase (Salusjärvi et al. 2017).

None of the recombinant strains designed in the present study could sustain growth on D-xylose alone and only D-xylonate formation was detected. Although all genes were transcribed, it remained unclear whether the corresponding proteins were catalytically active and functional in S. cerevisiae since only the activity of the first enzyme XylB could be verified. The combined assay of the three last enzymes (XylD, XylX, XylA) as well as the XylD (TBA) assay gave no activity in any of the recombinant $S$. cerevisiae strains. Due to the lack of products downstream of $\mathrm{D}$-xylonate, it is likely that the dehydration of D-xylonate to 2-keto-3-deoxy-D-xylonate, facilitated by the iron-sulfur $(\mathrm{Fe}-\mathrm{S})$ cluster containing protein XylD, was a major bottleneck to attain a fully active Weimberg pathway. Other studies on introduction of the Weimberg pathway into Pseudomonas species and C. glutamicum also showed D-xylonate accumulation during cultivations, and its conversion into 2-keto3-deoxy-D-xylonate occurred only when other carbon sources were depleted. Therefore, the reaction catalyzed by XylD was seemingly the rate-limiting step for an efficient $\mathrm{D}$-xylose oxidation into $\alpha$-ketoglutarate, also in these bacterial hosts (Meijnen et al. 2009; Radek et al. 2014). However, it cannot be ruled out that the second dehydratase in the Weimberg pathway, XylX, which catalyzes the dehydration of 2-keto-3-deoxy-D-xylonate to $\alpha$-ketoglutarate semialdehyde may also not be functional. This enzyme is not a $\mathrm{Fe}-\mathrm{S}$ cluster containing protein and protein BLAST analysis (NCBI: National Center for Biotechnology Information) reveals that the first 122 amino acids of the XylX protein (total length, 140 amino acids) contain a conserved domain from the fumarylacetoacetate (FAA) hydrolase family protein that is part of the MhpD superfamily of proteins. Further insights into the functionality of the Weimberg pathway could be revealed by analysis of its intracellular metabolites. However, this is currently hampered by the lack of commercial standards.

A recent study on in depth characterisation of $C c$ XylD heterologously expressed in E. coli led to the identification of three conserved cysteine residues that coordinate the $\mathrm{Fe}-\mathrm{S}$ binding essential for catalytic activity of the protein (Andberg et al. 2016). Although the formation of $\mathrm{Fe}-\mathrm{S}$ clusters occurs spontaneously in vitro, systems for $\mathrm{Fe}-\mathrm{S}$ biogenesis are required in vivo (Roche et al. 2013). In eukaryotes, the assembly of iron-sulfur clusters is 
carried out by two complex machineries, one present in the mitochondria (ISC: mitochondrial iron-sulfur cluster) and one present in the cytosol (CIA: cytosolic iron-sulfur protein assembly) (Lill et al. 2015; Lill and Mühlenhoff 2008). These machineries assemble $\mathrm{Fe}-\mathrm{S}$ clusters on scaffold proteins using iron and cysteine as the source of sulfur, and the Fe-S cluster is then transferred to apoproteins with the aid of specific chaperones (Lill and Mühlenhoff 2008). The chances of a correct formation of bacterial Fe-S cluster harboring proteins in $S$. cerevisiae remains unknown, since the biogenesis systems are significantly different in prokaryotes and eukaryotes (Kirby et al. 2016). However, examples of challenging functional expression of bacterial genes encoding $\mathrm{Fe}-\mathrm{S}$ cluster harboring enzymes in eukaryotes have been reported. For instance, the prokaryotic 6-phosphogluconate dehydratase (PGDH) from $E$. coli is a Fe-S clusterharboring enzyme, and despite a number of attempts to activate the $\mathrm{Fe}-\mathrm{S}$ cluster machinery in yeast by increasing the intracellular iron availability, inducing iron-responsive genes or by localizing PGDH to the mitochondria, the activity and functionality of PGDH could not be improved (Benisch and Boles 2014). An insufficient loading of the $\mathrm{Fe}-\mathrm{S}$ cluster onto the protein was hypothesized to result in a very low enzyme activity, since the $\mathrm{Fe}-\mathrm{S}$ cluster machinery in S. cerevisiae failed to recognize it as an apoprotein (Benisch and Boles 2014). In a similar line, the activity of the bacterial enzyme IspG, whose activity is also dependent on the correct assembly of Fe-S clusters, was demonstrated to be the limiting step to obtain a functional heterologous pathway for the production of isoprenoids in S. cerevisiae (Carlsen et al. 2013; Kirby et al. 2016). In our case, all Fe-S XylD homologs were detected, indicating again that the translation and folding of the proteins may not be the problem, whereas the proper loading of the Fe-S cluster might be.

In an attempt to circumvent the $\mathrm{Fe}-\mathrm{S}$ cluster problem, we searched for non Fe-S XylD homologs. In previous work focusing on enolase dehydratases, several six carbon dehydratases using D-mannonate or D-gluconate as substrate has been reported (Wichelecki et al. 2014) but no bacterial enzyme that can dehydrate D-xylonate has yet been found [reviewed by (Gerlt et al. 2012)]. Reported non $\mathrm{Fe}-\mathrm{S}$ XylD come from extremophilic archaea such as the halophilic H. volcanii (Johnsen et al. 2009) and the thermophilic Sulfolobus solfataricus (Nunn et al. 2010). Based on the lower temperature optimum of $H$. volcanii $\left(42{ }^{\circ} \mathrm{C}\right)$ it was chosen over the thermophilic $S$. solfataricus $\left(75-85^{\circ} \mathrm{C}\right)$ for this study. Thus, xad from $H$. volcanii ( $\left.x a d_{-} H v\right)$ was expressed in S. cerevisiae together with $x y l B, x y l X$ and $x y l A$ from $C$. crescentus (strain TMB4531). The use of $H v$ XAD in TMB4531 increased the uptake and formation rates of D-xylose and D-xylonate, respectively, which could indicate some flux downstream of D-xylonate. Supporting this is the fact that the carbon recovery in the fed-batch experiment with D-xylose and D-glucose was $85 \%$ for TMB4531, while for TMB4530 harboring XylD from C. crescentus it was $100 \%$. However, no further intermediates of the Weimberg pathway could be detected and no peptides corresponding to XAD could be found in TMB4531. Therefore, it is more likely that measurement error contributed to this result seen in the bioreactor experiment. Functional expression of genes originating from halophiles such as $\mathrm{H}$. volcanii can be challenging since these organisms require $100-150 \mathrm{~g} \mathrm{~L}^{-1}$ salt to grow and maintain structural stability. The proteome of halophiles is usually acidic leading to protein denaturation when suspended in low salt (Oren 2008). Thus, the absence of XAD in S. cerevisiae strain TMB4531 could indicate that the protein folding of this halophilic protein was indeed problematic, and the increased D-xylose bioconversion rate observed in this strain in comparison to TMB4530 was probably caused by an increased metabolic burden in TMB4530 expressing and assembling the Fe-S cluster-containing XylD.

In conclusion, the dehydration reaction(s) appear to be major bottlenecks to establish an active Weimberg pathway in S. cerevisiae. Bacterial XylDs require assembly of $\mathrm{Fe}-\mathrm{S}$ clusters for a functional active enzyme while archaeal XylDs of the enolase family originating from halophiles require high salt concentration for correct folding. At the time of writing, the hypothesis of a non- or poorly functional XylD was confirmed in a study where the Fe-S cluster metabolism of $S$. cerevisiae was altered by deletion of $F R A 2$ that regulates iron regulon transcription (Salusjärvi et al. 2017). This resulted in an increased activity of XylD from C. crescentus in yeast. Moreover, the XylD activity was improved when its expression was targeted to the mitochondrium, indicating that the cytosolic $\mathrm{Fe}-\mathrm{S}$ cluster synthesis was inefficient in yeast (Salusjärvi et al. 2017). Further evaluation of this strategy as well as alternative D-xylonate dehydratases with different requirements and properties, is therefore expected to lead to significant improvements of Weimberg functionality in yeast. Similarly, the crystallization of $H$. volcanii XAD could open new routes for optimization of this protein functionality through protein engineering.

\section{Additional files}

Additional file 1: Table S1. Primers used in the present study.

Additional file 2: Figure S1. Schematic representation of the integration of the Weimberg pathway encoding genes into S. cerevisiae. The CRISPR-Cas9 system and nested homologous recombination was used to integrate $x y|B, x y| X, x y \mid A$ together with $x y \mid D$ or $x a d$ from plasmid $p A G S 8 B$ or PAGS8HB, respectively, replacing the GRE3 ORF (panel A). This resulted in 
strain TMB4530 ( $x y|B, x y| D, x y \mid X$ and $x y \mid A)$ and strain TMB4531 ( $x y|B, x a d, x y| X$ and $x y / A$ ). The 20 bp gRNA sequence used to target the Cas 9 nuclease to the GRE3 ORF is shown in bold. The CRISPR-Cas9 system was also used to replace xyID from Caulobacter crescentus in TMB4530 with three different homologs from Burkholderia cenocepacia, Escherichia coli and Ellin329 isolate generating strains TMB4569, TMB4570 and TMB4571, respectively (Panel B). The 20 bp gRNA sequence used to target the Cas9 nuclease to the $C$. crescentus xyID in TMB4530 is shown in bold.

Additional file 3: Figure S2. UHPLC and HPLC chromatograms of end point samples from the bioreactor experiments. Panel $A$ and $B$ show UHPLC chromatograms for strain TMB4530 (A) and TMB4531 (B) at the end of the fermentation experiment. No unidentified peaks could be found indicating that no detectable amounts of Weimberg intermediates were produced. Panel C and D show HPLC chromatograms for strain TMB4530 (C) and TMB4531 (D) from the same sample. One extra small peak can be seen (medium component), which was present at the start of fermentation thereby eliminating the possibility that this peak contains Weimberg intermediates

\section{Abbreviations}

AGE: agarose gel electrophoresis; AKG: a-ketoglutarate; Bc: Burkholderia cenocepacia; CDW: cell dry weight; CIA: cytosolic iron-sulfur protein assembly; Ec: Escherichia coli; El: Ellin329 isolate; HPLC: high-performance liquid chromatography; Hv: Haloferax volcanii; ISC: mitochondrial iron-sulfur cluster; LB: Luria-Bertani broth; $\mathrm{NAD}^{+} / \mathrm{H}$ : nicotinamide adenine dinucleotide; PCR: polymerase chain reaction; PGDH: 6-phosphogluconate; PPP: pentose phosphate pathway; SM: synthetic media; TCA cycle: tricarboxylic acid cycle; UHPLC: ultra-performance liquid chromatography; XDH: D-xylitol dehydrogenase; XI: D-xylose isomerase; XR: D-xylose reductase; YNB: yeast nitrogen base; YPD: yeast peptone dextrose media; Y-PER: yeast protein extraction reagent.

\section{Authors' contributions}

LW and AGS participated in the design of the study and its coordination, carried out molecular genetic engineering and strain constructions. LW and DPN carried out growth assays, HPLC analysis and drafted the manuscript. HA carried out bioreactor experiments, UPLC and HPLC analysis. MGG and GL conceived the study, participated in the design of the study and its coordination and helped to draft the manuscript. All authors read and approved the final manuscript.

\section{Author details}

1 Division of Applied Microbiology, Department of Chemistry, Lund University, PO Box 124, 22100 Lund, Sweden. ${ }^{2}$ Department of Chemical Engineering, Lund University, PO Box 124, 22100 Lund, Sweden. ${ }^{3}$ Present Address: Harboes Bryggeri A/S, Spegerborgvej 34, 4230 Skælskør, Denmark. ${ }^{4}$ Present Address: Novozymes A/S, Krogshøjvej 36, 2880 Bagsværd, Denmark.

\section{Acknowledgements}

We would like to thank all the partners in the EU consortium project BioREFINE-2G, in particular Irina Borodina and Vratislav Stovicek for sharing their CRISPR-Cas9 system and for giving valuable input. Nina Bjurman and Alexander Rex are thanked for excellent technical support. In addition, special thanks to Stephan Noack and Bianca Klein from the ERASysAPP XyloCut project for performing the proteomics analysis on XyID protein at Jülich Forschungszentrum. The EU commission (FP7 BioREFINE-2G, Project Number 613771) is acknowledged for financial support.

\section{Competing interests}

The authors declare that they have no competing interests.

\section{Availability of data and materials}

Raw data and materials will be shared upon request.

\section{Consent for publication}

Not applicable.

\section{Ethics approval and consent to participate} Not applicable.

\section{Funding}

This work was supported by the European Union Commission (FP7 BioREFINE2G, Project No. 613771).

\section{Publisher's Note}

Springer Nature remains neutral with regard to jurisdictional claims in published maps and institutional affiliations.

Received: 4 December 2017 Accepted: 28 February 2018 Published online: 05 March 2018

\section{References}

Abbott DA, Zelle RM, Pronk JT, Van Maris AJ (2009) Metabolic engineering of Saccharomyces cerevisiae for production of carboxylic acids: current status and challenges. FEMS Yeast Res 9:1123-1136

Almqvist H, Sandahl M, Liden G (2017) A rapid method for analysis of fermentatively produced $\mathrm{D}$-xylonate using ultra-high performance liquid chromatography and evaporative light scattering detection. Biosci Biotechnol Biochem 81:1078-1080

Andberg M, Aro-Kärkkäinen N, Carlson P, Oja M, Bozonnet S, Toivari M, Hakulinen N, O'Donohue M, Penttilä M, Koivula A (2016) Characterization and mutagenesis of two novel iron-sulphur cluster pentonate dehydratases. Appl Microbiol Biotechnol 100:7549-7563

Aurich A, Specht R, Müller RA, Stottmeister U, Yovkova V, Otto C, Holz M, Barth G, Heretsch P, Thomas FA (2012) Microbiologically produced carboxylic acids used as building blocks in organic synthesis. In: reprogramming microbial metabolic pathways. Springer, Berlin, pp 391-423

Bajaj I, Singhal R (2011) Poly (glutamic acid)—an emerging biopolymer of commercial interest. Biores Technol 102:5551-5561. https://doi. org/10.1016/j.biortech.2011.02.047

Benisch F, Boles E (2014) The bacterial Entner-Doudoroff pathway does not replace glycolysis in Saccharomyces cerevisiae due to the lack of activity of iron-sulfur cluster enzyme 6-phosphogluconate dehydratase. J Biotechnol 171:45-55

Borodina I, Nielsen J (2014) Advances in metabolic engineering of yeast Saccharomyces cerevisiae for production of chemicals. Biotechnol I 9:609-620

Bradford MM (1976) A rapid and sensitive method for the quantitation of microgram quantities of protein utilizing the principle of protein-dye binding. Anal Biochem 72:248-254

Buchanan CL, Connaris H, Danson MJ, Reeve CD, Hough DW (1999) An extremely thermostable aldolase from Sulfolobus solfataricus with specificity for non-phosphorylated substrates. Biochem J 343(Pt 3):563-570

Buchert J, Viikari L (1988) The role of xylonolactone in xylonic acid production by Pseudomonas fragi. Appl Microbiol Biotechnol 27:333-336

Buijs NA, Siewers V, Nielsen J (2013) Advanced biofuel production by the yeast Saccharomyces cerevisiae. Curr Opin Chem Biol 17:480-488

Carlsen S, Ajikumar PK, Formenti LR, Zhou K, Phon TH, Nielsen ML, Lantz AE, Kielland-Brandt MC, Stephanopoulos G (2013) Heterologous expression and characterization of bacterial 2-C-methyl-D-erythritol-4-phosphate pathway in Saccharomyces cerevisiae. Appl Microbiol Biotechnol 97:5753-5769

Djurdjevic l, Zelder O, Buckel W (2011) Production of glutaconic acid in a recombinant Escherichia coli strain. Appl Environ Microbiol 77:320-322

Du Preez J, Prior B (1985) A quantitative screening of some xylose-fermenting yeast isolates. Biotech Lett 7:241-246

Dutta S, Ray S, Nagarajan K (2013) Glutamic acid as anticancer agent: an overview. Saudi Pharm J 21:337-343

Entian KD, Kötter P (2007) Yeast genetic strain and plasmid collections. Methods Microbiology 36:629-666

Faller LD, Baroudy BM, Johnson AM, Ewall RX (1977) Magnesium ion requirements for yeast enolase activity. Biochemistry 16:3864-3869

Fink J (2013) Reactive polymers fundamentals and applications, vol 2. William Andrew, Norwich

Gerlt JA, Babbitt PC, Jacobson MP, Almo SC (2012) Divergent evolution in enolase superfamily: strategies for assigning functions. J Biol Chem 287:29-34

Gietz RD, Schiestl RH (2007) High-efficiency yeast transformation using the LiAc/SS carrier DNA/PEG method. Nat Protoc 2:31-34 
Hahn-Hägerdal B, Karhumaa K, Jeppsson M, Gorwa-Grauslund MF (2007) Metabolic engineering for pentose utilization in Saccharomyces cerevisiae. In: Biofuels. Springer, Berlin, pp 147-177

Heigwer F, Kerr G, Boutros M (2014) E-CRISP: fast CRISPR target site identification. Nat Methods 11:122-123

Jeffries TW (1983) Utilization of xylose by bacteria, yeasts, and fungi. In: Pentoses and Lignin. Springer, Berlin, pp 1-32

Jin Y-S, Laplaza JM, Jeffries TW (2004) Saccharomyces cerevisiae engineered for xylose metabolism exhibits a respiratory response. Appl Environ Microbiol 70:6816-6825

Johnsen U, Dambeck M, Zaiss H, Fuhrer T, Soppa J, Sauer U, Schönheit P (2009) D-Xylose degradation pathway in the halophilic archaeon Haloferax volcanii. J Biol Chem 284:27290-27303

Kim SR, Park Y-C, Jin Y-S, Seo J-H (2013) Strain engineering of Saccharomyces cerevisiae for enhanced xylose metabolism. Biotechnol Adv 31:851-861

Kirby J, Dietzel KL, Wichmann G, Chan R, Antipov E, Moss N, Baidoo EE, Jackson P, Gaucher SP, Gottlieb S (2016) Engineering a functional 1-deoxy-D-xylulose 5-phosphate (DXP) pathway in Saccharomyces cerevisiae. Metab Eng 38:494-503

Lien O Jr (1959) Determination of gluconolactone, galactonolactone, and their free acids by hydroxamate method. Anal Chem 31:1363-1366

Lill R, Mühlenhoff U (2008) Maturation of iron-sulfur proteins in eukaryotes: mechanisms, connected processes, and diseases. Annu Rev Biochem 77:669-700

Lill R, Dutkiewicz R, Freibert SA, Heidenreich T, Mascarenhas J, Netz DJ, Paul VD, Pierik AJ, Richter N, Stümpfig M (2015) The role of mitochondria and the CIA machinery in the maturation of cytosolic and nuclear iron-sulfur proteins. Eur J Cell Biol 94:280-291

Lõoke M, Kristjuhan K, Kristjuhan A (2011) Extraction of genomic DNA from yeasts for PCR-based applications. Biotechniques 50:325

Markowitz VM, Chen IM, Palaniappan K, Chu K, Szeto E, Pillay M, Ratner A, Huang J, Woyke T, Huntemann M, Anderson I, Billis K, Varghese N, Mavromatis K, Pati A, Ivanova NN, Kyrpides NC (2014) IMG 4 version of the integrated microbial genomes comparative analysis system. Nucleic Acids Res 42:D560-D567

McWilliam H, Li W, Uludag M, Squizzato S, Park YM, Buso N, Cowley AP, Lopez R (2013) Analysis tool web services from the EMBL-EBI. Nucleic Acids Res 41:W597-W600

Meijnen J-P, de Winde JH, Ruijssenaars HJ (2009) Establishment of oxidative D-xylose metabolism in Pseudomonas putida S12. Appl Environ Microbiol 75:2784-2791

Nunn CE, Johnsen U, Schonheit P, Fuhrer T, Sauer U, Hough DW, Danson MJ (2010) Metabolism of pentose sugars in the hyperthermophilic archaea Sulfolobus solfataricus and Sulfolobus acidocaldarius. J Biol Chem 285:33701-33709

Nygård Y, Maaheimo H, Mojzita D, Toivari M, Wiebe M, Resnekov O, Pesce CG, Ruohonen L, Penttilä M (2014) Single cell and in vivo analyses elucidate the effect of xylC lactonase during production of D-xylonate in Saccharomyces cerevisiae. Metab Eng 25:238-247

Oren A (2008) Microbial life at high salt concentrations: phylogenetic and metabolic diversity. Saline Syst 4:2

Otto C, Yovkova V, Barth G (2011) Overproduction and secretion of a-ketoglutaric acid by microorganisms. Appl Microbiol Biotechnol 92:689-695

Paddon CJ, Westfall P, Pitera D, Benjamin K, Fisher K, McPhee D, Leavell M, Tai A Main A, Eng D (2013) High-level semi-synthetic production of the potent antimalarial artemisinin. Nature 496:528-532
Petsko GA, Ringe D (2004) Protein structure and function. New Science Press, London

Radek A, Krumbach K, Gätgens J, Wendisch VF, Wiechert W, Bott M, Noack S, Marienhagen J (2014) Engineering of Corynebacterium glutamicum for minimized carbon loss during utilization of D-xylose containing substrates. J Biotechnol 192:156-160

Roche B, Aussel L, Ezraty B, Mandin P, Py B, Barras F (2013) Iron/sulfur proteins biogenesis in prokaryotes: formation, regulation and diversity. Biochem Biophys Acta 1827:923-937

Ronda C, Pedersen LE, Hansen HG, Kallehauge TB, Betenbaugh MJ, Nielsen AT, Kildegaard HF (2014) Accelerating genome editing in CHO cells using CRISPR Cas9 and CRISPy, a web-based target finding tool. Biotechnol Bioeng 111:1604-1616

Sailakshmi G, Mitra T, Chatterjee S, Gnanamani A (2013) Chemistry behind the elastic nature of the biomaterial prepared using oxidized form of glutaraldehyde and chitosan — an approach at 2D and 3D Level. Int J Life Sci Med Res 4:64-75

Salusjärvi L, Toivari M, Vehkomaki ML, Koivistoinen O, Mojzita D, Niemela K Penttilä M, Ruohonen L (2017) Production of ethylene glycol or glycolic acid from D-xylose in Saccharomyces cerevisiae. Appl Microbiol Biotechnol 101:8151-8163

Solis-Escalante D, Kuijpers NG, Nadine B, Bolat I, Bosman L, Pronk JT, Daran J-M, Pascale D-L (2013) amdSYM, a new dominant recyclable marker cassette for Saccharomyces cerevisiae. FEMS Yeast Res 13:126-139

Stephens C, Christen B, Fuchs T, Sundaram V, Watanabe K, Jenal U (2007) Genetic analysis of a novel pathway for D-xylose metabolism in Caulobacter crescentus. J Bacteriol 189:2181-2185

Stovicek V, Borodina I, Forster J (2015) CRISPR-Cas system enables fast and simple genome editing of industrial Saccharomyces cerevisiae strains. Metab Eng Commun 2:13-22

Sung MH, Park C, Kim CJ, Poo H, Soda K, Ashiuchi M (2005) Natural and edible biopolymer poly-gamma-glutamic acid: synthesis, production, and applications. Chem Rec 5:352-366

Toivari MH, Ruohonen L, Richard P, Penttilä M, Wiebe MG (2010) Saccharomyces cerevisiae engineered to produce D-xylonate. Appl Microbiol Biotechnol 88:751-760

Toivari M, Nygård Y, Kumpula E-P, Vehkomäki M-L, Benčina M, Valkonen M, Maaheimo H, Andberg M, Koivula A, Ruohonen L (2012) Metabolic engineering of Saccharomyces cerevisiae for bioconversion of D-xylose to D-xylonate. Metab Eng 14:427-436

Van Maris AJ, Winkler AA, Kuyper M, De Laat WT, Van Dijken JP, Pronk JT (2007) Development of efficient xylose fermentation in Saccharomyces cerevisiae: xylose isomerase as a key component. In: Biofuels. Springer, Berlin, pp 179-204

Wang PY, Schneider H (1980) Growth of yeasts on D-xylulose. Can J Microbiol 26:1165-1168

Weimberg R (1961) Pentose oxidation by Pseudomonas fragi. J Biol Chem 236:629-635

Wichelecki DJ, Balthazor BM, Chau AC, Vetting MW, Fedorov AA, Fedorov EV, Lukk T, Patskovsky YV, Stead MB, Hillerich BS, Seidel RD, Almo SC, Gerlt JA (2014) Discovery of function in the enolase superfamily: D-mannonate and D-gluconate dehydratases in the D-mannonate dehydratase subgroup. Biochemistry 53:2722-2731

\section{Submit your manuscript to a SpringerOpen ${ }^{\circ}$ journal and benefit from:}

- Convenient online submission

- Rigorous peer review

- Open access: articles freely available online

- High visibility within the field

Retaining the copyright to your article

Submit your next manuscript at $\boldsymbol{~ s p r i n g e r o p e n . c o m ~}$ 